\title{
BILINGUES BRUTTACES. IL PLURILINGUISMO DI UNA CITTÀ DELLA MAGNA GRECIA ATTRAVERSO I SUOI TESTI: IL CASO DI PETELIA
}

\author{
PAOLO POCCETTI $(*)$
}

RIASSUNTO. - Una nuova tabella defixionis dal Bruzio suggerisce nuovi spunti di riflessione sui fenomeni del bilinguismo osco-greco nella Calabria antica. A partire da questo testo, peraltro molto problematico, si cercherà di analizzare quanto può ascriversi al contesto specifico che l'ha prodotto, quanto è riferibile all'ambiente regionale e, infine, quanto ha ricadute a maglia più larga nell'ambito del greco, del latino fino agli sviluppi romanzi.

$* * *$

ABSTRACT. - The osco-greek bilingualism in a Bruzio town: Petelia. In the light of both epigraphic and literary texts.

A new tabella defixionis from Bruzio suggests new points of reflection on the phenomena of the osco-Greek bilingualism in ancient Calabria. Basing on this very problematic text, we'll try to analyze how much it can be ascribed to the specific context that has produced him, how much it is referable to the regional environment and, finally, how much it has reverted in a larger network in the circle of Greek and Latin up to the romance development.

\section{PREMESSA}

Tra le città della Magna Grecia l'antico insediamento noto dalle fonti sotto il nome di Petelia, situato sul versante ionico della Calabria in prossimità dell'odierna Strongoli, nell'antico territorio crotoniate, presenta un dossier epigrafico tra i più interessanti e signi-

(*) Università degli Studi di Roma, Tor Vergata. 
ficativi per la sua varietà in senso sincronico e diacronico nell'ambito della Magna Grecia. Pur nel suo carattere frammentario e desultorio, la documentazione di Petelia offre uno straordinario squarcio trasversale del plurilinguismo della città e della sua distribuzione sociolinguistica, snodandosi lungo un filo che annoda i momenti e i fatti salienti della storia linguistica di questo sito. Ci sembra, pertanto, utile presentare la raccolta delle testimonianze epigrafiche e letterarie al fine di delineare attraverso i tasselli dei testi il complesso mosaico linguistico.

La sollecitazione a riprendere in esame l'intero dossier documentario è scaturita dalla recente pubblicazione di due testi, caratterizzati da spiccati fenomeni di contatto linguistico, l'uno tra osco e greco $\left(\mathrm{n}^{\circ} 5\right)$, l'altro tra greco e latino $\left(\mathrm{n}^{\circ} 11\right)$.

L'insieme di questa documentazione, come testimonianza dell'articolazione sociolinguistica del plurilinguismo, non è solo meritevole di interesse per il sito specifico, ma è anche in una certa misura paradigmatico in una prospettiva più ampia in rapporto alla regione di riferimento, la Calabria, per la quale i fenomeni di contatto e di continuità tra lingue sono stati da tempo oggetto di dibattito. Tuttavia, la peculiarità di questi fenomeni su scala regionale era già ben presente alla coscienza linguistica del mondo antico, di cui è emblematica la definizione bilingues Bruttaces attribuita ad Ennio e Lucilio e glossata da Festo quod Bruttii et Osce et Graece loqui soliti sint (testi nn.1a,b). ${ }^{1}$ Del mosaico dei bilingues Bruttaces i testi di Petelia costituiscono tasselli documentari di prim'ordine.

\section{INQUADRAMENTO STORICO}

Petelia è il nome antico del sito ubicato nella fascia costiera ionica compresa tra il territorio di due antiche colonie achee, quello di Sibari e quello di Crotone. Nella tradizione antica, recepita da Strabone, Petelia è considerata, come altre città del versante ionico della Calabria, fondazione di Filottete e, dunque, legata attraverso il mondo dei nostoi, alle più antiche frequentazioni dei Greci in

1 P. Fest 31 L. = Enn., ann. 496 Vahlen ; Porph. Ad Hor., Sat. I 10, 30 = Lucil. 1124 Marx. 
Occidente $\left(\mathrm{n}^{\circ} 2\right){ }^{2}$ Tuttavia, come notava già H.Krahe, ${ }^{3}$ il toponimo è probabile travestimento greco (forse per associazione paretimologica a peta $\mid$ oj / peth| oj ) di un nome locale anellenico. Dei contatti antichissimi con il mondo indigeno si serba traccia anche nella tradizione sulla figura di Filottete, di cui è stata messa in evidenza la 'connotazione ambigua'4 per quanto attiene il rapporto tra Greci e le genti autoctone. Infatti, in questo contesto Filottete appare come un eroe greco che intrattiene legami di amicizia con le popolazioni anelleniche, ma che, allo stesso tempo, muore per mano di quest'ultime. Inoltre, anche sul versante greco, Filottete sembra legare tra loro diversi ambienti. La leggenda delle sue imprese è, infatti, connessa al circuito delle colonie achee, in particolare, a Crotone, che ne è stato uno dei principali vettori, ma è anche rivendicato da altri ambiti 'coloniali', come quello dei Rodii e quello 'ateniese' di Thurii.'

Alla base dei diversi filoni confluiti nelle fonti per quanto riguarda i rapporti di Filottete con il mondo indigeno si colgono fasi alterne ed atteggiamenti diversi che le singole realtà indigene hanno avuto con la grecità, soprattutto in insediamenti, per così dire, 'di frontiera' o situati al margine tra i territori di due importanti colonie achee come Sibari e Crotone. L'evoluzione delle rispettive storie insieme a quella, in parte parallela, in parte intersecantesi, delle popolazioni anelleniche si riverbera nell'intreccio delle diverse tradizioni leggendarie che costituiscono lo sfondo storico nel quale si colloca il sito di Petelia. Infatti, la stessa notizia straboniana che rivendica la grecità di Petelia attribuendola ad una fondazione di Filottete ne riconosce anche due ruoli distinti, probabilmente scaglionati su due diverse fasi cronologiche, dal punto di vista delle presenze indigene, segnalandolo, da una parte, come 'luogo fortificato' dai Sanniti (elumnh/Sa unika i pote frouribij epeteikis a n a uth h) e, dall'altra come mhtropol ij tw L euka nw \#). Infine, circostanza che ha un interesse sincronico agli occhi di Strabone è il fatto che all'epoca augustea il sito era ancora abbastanza abitato (s unoik ei ka i mek ri nu $\mathrm{i} k$ a nw $F$ ), a differenza di altre città assegnate a fondazioni di Filottete, quali Krimissa e Makalla, o di siti scomparsi della

2 Strab. VI 1, 3254 C.

3 Cfr. Krahe 1941, p. 138.

4 Così Giangiulio 1991, p. 51.

5 Cfr. Mele 2007, 44 ss; 68 ss. 
Lucania, quali 0 u ert i fa i e K a I a $\$$ a r na ${ }^{6}$ di cui, al dire del geografo augusteo, non restava altro che l'eco lontana del nome. Tale dato ci viene confermato dalle iscrizioni che accompagnano la vita della città fino all'avanzata età imperiale.

Per l'epoca pre-romana, invece, il dettato di Strabone pone una questione di più delicata attinenza alla topografia storica, ma che ha anche qualche ricaduta linguistica, cioè le presenze di Lucani e di Sanniti nella città, che è ubicata nella regione dei Bruzi. Due ne sono gli aspetti nodali: a) la qualifica di Petelia come 'Lucana' rispetto alla sua ubicazione topografica nell'ager Bruttius (almeno a partire dal costituirsi dei Bruzi come entità regionale autonoma, fissata dalle fonti nel 356 a.C.); b) l'accenno alle presenze di 'Sanniti' in quel contesto.

Nella notizia di Strabone il riferimento ai Lucani e ai Sanniti in relazione a Petelia sembra ripartirsi tra due ruoli diversi. Ai Lucani si lega la definizione della città come mhtropol ij tw L eukanw che si pone in parallelo a quella attribuita, sempre da Strabone, a Cosenza come mhtropol ij tw B B ettiwn, mentre i Sanniti sono chiamati in causa per il loro intervento nella costituzione del sito come centro fortificato. In base a tale distinzione il legame con i Lucani sembra piuttosto di tipo ideologico, se si attribuisce a mht ropol ij il valore di 'centro o modello di irradiazione', mentre ai Sanniti si collega una tipologia insediativa, incardinata su una struttura fortificata di caratteristiche tipicamente italiche, se si fa corrispondere il termine greco frourion alla nozione (anche istituzionale) di ocri- nella cultura italica. ${ }^{8}$

D'altra parte anche la diversità dei ruoli a cui sono connessi i due etnonimi menzionati da Strabone a proposito di Petelia non esclude una loro differente scansione cronologica. Particolarmente problematica è la dizione mhtropol ij tw L eukanwF, che ha giustamente sollevato la questione se «ha un fondamento scientifico la nostra comune convinzione che Petelia fosse già diventata brettia a partire dal 357/56 a.C.».' Tale domanda è stata assoggettata a due livelli di spiegazione diversi. L'uno, in chiave più strettamente cronologica, si incardina sulla visione di una 'grande Lucania' antecedente alla fase in cui i Bruzi ancora non si erano costi-

6 Strab. VI 1, 3, 254 C.

7 Strab. VI 1, 5256 C.

8 Cfr. Prosdocimi 1978, p. 38 ss.

9 Citazione da De Sensi Sestito 2004, p. 551. 
tuiti come realtà regionale, i cui confini, secondo Strabone, coinciderebbero con quelli dell' 'It a I i i di Antioco, segnati dalla linea che va da Laos sul Tirreno a Metaponto sullo Ionio. ${ }^{10}$ Pertanto, la visione di 'Petelia lucana' dovrebbe rispecchiare una situazione antecedente l'evento datato dalle fonti al 356 a.C. ${ }^{11}$ L'altro, invece, facendo leva sulla fluidità del confine con i Bruzi sul versante ionico e sulle difficoltà di distinguere, soprattutto in quella fascia costiera, indicatori archeologici 'lucani' da quelli 'bruzi'12 è portato a considerare «Petelia città romana a tutti gli effetti fino a Pirro». ${ }^{13}$

E' lecito domandarsi se il corpus di testi disponibile può fornire un contributo alla questione lasciata aperta dal dettato straboniano. Tale domanda si inserisce nel solco di una ricerca, che, ormai oltre un quarto di secolo $\mathrm{fa},{ }^{14}$ si era orientata verso la definizione di una identitità culturale che permettesse di distinguere in seno agli 'Italici del sud' i Bruzi dai Lucani dopo la scissione del 356 a.C., accadimento che segna l'inizio delle loro individualità etniche, secondo la nota scansione straboniana. La riflessione, che qui si presenta, si propone, da una parte, come sviluppo di quel modello di ricerca, concentrandosi, questa volta, in una dimensione 'sintopica' osservata lungo il suo asse diacronico.

\section{LA DOCUMENTAZIONE EPIGRAFICA}

Il più antico documento epigrafico attribuito a Petelia ${ }^{15}$ è la tabella di bronzo datata tra la fine del VI e l'inizio del V secolo, che contiene un atto di donazione $\left(\mathrm{n}^{\circ} 3\right)$. Il documento è scritto nella lingua e nell'alfabeto delle colonie achee e come tale viene inserito nelle raccolte epigrafiche sotto la pertinenza al territorio di Crotone. ${ }^{16}$

10 Strab. VI 1, 4254 C.

11 Cfr. Musti 1994, p. 272 ss.

12 Cfr. Guzzo 1988, 179 ss.

13 Così De Sensi Sestito 2004, p. 553.

14 Poccetti 1988.

15 Il testo, generalmente attribuito a Petelia, è frutto di un rinvenimento settecentesco, dove appare anche la menzione di Policastro. Per una riconsiderazione dei dati di rinvenimento che mettono in dubbio la provenienza dal sito dell'antica Petelia cfr. Granei 1995.

16 Cfr. IG XIV 636; Arena 1996, n 51 (dove si dà una datazione tra fine VI e inizio V); Dubois 2002, $\mathrm{n}^{\circ} 93$ (dove si data all'inizio V). 
Tale documento appartiene ad una tipologia ben nota in Magna Grecia in età tardo-arcaica, quella del lascito testamentario. ${ }^{17}$ Nella fattispecie il testo registra un certo (o una certa) $\mathrm{S}$ a $0 \mathrm{ij}^{18}$ che dona a una donna di nome S ika ini $\operatorname{a}$ la casa e tutti i suoi averi. L'identificazione del donatore con una figura maschile a fronte di quella femminile della beneficiaria trova parallelo in un'altra lamina della stessa area che ricorda il lascito da parte di un uomo alla propria moglie ${ }^{19}$. La lamina attribuita a Petelia condivide, oltre alle peculiarità dialettali e la similarità del formulario, due fatti istituzionali specifici che la collegano più direttamente alle colonie achee di Sibari e di Crotone, l'eponimia del da mi ou r gol' ricorrente nell'epigrafia crotoniate,$^{20}$ e l'uso del termine prokenoj con il valore di 'testimone', ${ }^{21}$ che si presenta anche nel trattato tra Sibariti e Serdaioi ritrovato ad Olimpia. ${ }^{22}$ Invece, a Terina nella Calabria tirrenica, un documento di analogo tenore, sia pure più tardo, ${ }^{23}$ oltre alla differente struttura testuale, presenta un termine per 'testimone' diverso da p r o k e noj ${ }^{24} \mathrm{e}$ il riferimento ad altra carica pubblica. ${ }^{25}$

Il testo attribuito a Petelia rivela, dunque, una forte permeabilità al lessico istituzionale delle due colonie achee, Sibari e Crotone, nella cui orbita, del resto, il sito di rinvenimento gravitava. La menzione della carica del da mi ou rgol sembra accennare ad una più forte influenza istituzionale di Crotone, che sarà stata probabilmente esclusiva, se il documento è da datarsi dopo la caduta di Sibari nel 510 a.C.

Pertanto, il documento testamentario in questione mostra, al volgere del VI secolo, la piena partecipazione della città alle istituzioni di diritto privato e pubblico comuni ad altri siti della Magna Grecia, risentendo in modo più marcato del lessico giuridico ed istituzionale delle due colonie achee prossime, e, più marcatamente, di quello crotoniate. Merita, infine, sottolineare che la categoria testuale delle 'donazioni' a

17 Cfr. Arangio Ruiz-Olivieri 1925, nn.18-21 p. 143 s.

18 Per il carattere epiceno del nome cfr. Dubois 2002, p. 154.

19 Arena 1996, n52; Bencivenni 1997, n³; Dubois 2002, nº 100.

20 Cfr. Lazzarini 2003, p. 84.

21 Cfr. Dubois 2002, p. 155.

22 Arena 1996, n 3; Dubois 2002, $\mathrm{n}^{\circ} 12$.

23 Dubois 2002, $n^{\circ} 98$.

24 Si tratta probabilmente di epa k ooj : cfr. Granei 1999, p. 155.

25 Si tratta del pruta nij : cfr. Granei 1999, p. 152. 
cui appartiene la tabella di bronzo in questione ha una implicazione più generale per quanto riguarda il grado di alfabetizzazione, oltre che la diffusione e la gestione della scrittura a quella quota cronologica. Infatti, questo tipo di supporto, cioè le tabelle di bronzo, verosimilmente prevedeva anche luoghi deputati alla loro archiviazione e alla loro consultazione come documento giuridico che attesta il trasferimento della proprietà di beni immobili. ${ }^{26}$

Qualche sprazzo della variegata composizione sociale del territorio di Petelia già agli inizi del V secolo traluce dall'onomastica rispecchiata dal testo. Accanto ad antroponimi greci, piuttosto comuni, tra i quali spicca 'O na $\mathrm{ta} \mathrm{j}$, che è tra i nomi più frequenti nell'epigrafia delle colonie achee, ${ }^{27}$ si segnalano elementi non greci come $M i$ ink ōn, confrontato con le basi onomastiche di gentilizi latini come Mincius, Mi(n)culeius. ${ }^{28}$ Questione più complessa solleva il nome della beneficiaria della donazione, cioè Sika inila, difficilmente disgiungibile da S ik a ni a, nome della Sicilia, attestato fin da Omero. L'antroponimo femminile può spiegarsi in vari modi, che, tuttavia, rendono necessari interventi correttivi della forma epigraficamente attestata e cioè:

a) nome della Sicilia, usato in funzione di nome personale, come si verifica per la designazione concorrente $\mathrm{Si} \mathrm{k} \mathrm{el} \mathrm{i} \mathrm{A}$, attestato come nome individuale ad Atene, ${ }^{29}$ e, inversamente, per $\mathrm{K} \mathrm{hth}$ attestato come antroponimo a Sibari. ${ }^{30}$ In questo caso la forma tràdita necessita della correzione S ik a $\{\mathrm{i}\}$ nia ;

b) corrispondente femminile di Sikwn, connesso a sua volta al nome della Sicilia quale ipocoristico di Sik el of e Sika nol..$^{31}$ Sikaini a potrebbe essere stato rifatto su Sikwn secondo il modello $L$ a $k w n$ : $L$ a $k$ a ina.$^{32}$ Tale soluzione impone la correzione Sika in $\{i\} a$;

c) etnico (=S i k a na ) usato come antroponimo, che trova due corrispettivi (anche per omogeneità cronologica a Selinunte ${ }^{33}$ e a

\footnotetext{
26 Per questo aspetto cfr. Bencivenni 1997, p. 39.

27 Come sottolinea Arena 1996, p. 79.

28 Cfr. Dubois 2002, p. 156.

29 Cfr.Fraser-Matthews 1994, 397.

30 Cfr. Lazzarini 2000. Più prudente in questa interpretazione Dubois 2002, n³.

31 Cfr.Bechtel 1917, p.543; Wikén 1937, pp. 60, 69, 113; Trendall 1967, 213 :

32 Cfr. Dubois 2008, p. 155.

33 Dubois $1989, \mathrm{n}^{\circ} 29$.
} 
Camarina. ${ }^{34}$ In questo caso, però, gli emendamenti richiesti sarebbero più pesanti e cioè $S i k a\{i\} n\{i\} a .^{35}$

A proposito dei nomi della Sicilia impiegati come nomi personali vale la pena rammentare che, mentre in Grecia sono attestati S i k el oj e $S$ ik el $a b$ in Occidente in funzione di antroponimo non si trova che S i k a noj. Inoltre, due attestazioni su tre di quest'ultimo etnonimo, alle quali potrebbe aggiungersi quella attribuita a Petelia nell'ipotesi di lettura c) si riferiscono a donne. Parallelamente anche in Sicilia si trovano, oltre a S i ka na / altri nomi di donne derivati da etnici dell'Italia come L eFk a na ${ }^{6}{ }^{6}$ e T urra na /a Selinunte..$^{37}$

Il nome delle beneficiaria della donazione è, dunque, in qualche modo connesso al nome della Sicilia, anche se non implica una diretta provenienza della donna in questione dall'isola. Il dato resta comunque significativo dell'inserimento del sito di rinvenimento nel circuito di contatti e di mobilità personale tra le due sponde dello Stretto entro il quale l'uso di siffatto nome si colloca.

A Petelia si lega un altro importante documento dell'epigrafia della Magna Grecia, che denuncia le presenze di ambienti religiosi e culturali diffusi oltre i confini della Magna Grecia. Si tratta della lamina d'oro, rivenuta nel 1834 nella necropoli ed oggi conservata al British Museum $\left(n^{\circ} 4\right)^{38}$. Tale testo, appartenente al corpus delle lamine auree, che definiscono l'iniziazione dell'individuo in rapporto ad ambienti misterici e a credenze escatologiche, è datato alla prima metà del IV secolo, ${ }^{39}$ e, insieme a quello di Hipponion, antecedente di pochi decenni, è tra più antichi non solo tra quelli della grecità d'Occidente. Sul piano linguistico, il documento di Petelia condivide con le altre lamine l'uso del dialetto ionico legato al 'genere' nel quale viene veicolata la circolazione 'mediterranea' di motivi comuni a questi circoli iniziatici, distaccandosi, dunque, come, del resto, avviene in altri ambienti dalla cornice dialettale locale di impronta dorica. Sul piano formulare la

34 Cf. Dubois 1989, n¹20; Dubois 2008, n46,23.

35 Eccetto il caso c), dove la meccanica dell'errore è difficilmente spiegabile, nelle ipotesi a) e b) la dittografia di $<\mathbf{l}>$ potrebbe attribuirsi all'influsso della sillaba contigua.

\footnotetext{
36 Cf. Dubois $2008, \mathrm{n}^{\circ} 20$.

37 Dubois $1989, \mathrm{n}^{\circ} 37$.

38 IG XIV 638; Pugliese Carratelli 2001, p.67; Tortorelli Ghidini 2006, p.66.

39 Cfr. Pugliese Carratelli 2001, p.67; Tortorelli Ghidini 2006, p.66.
} 
lamina di Petelia si inserisce nella dialettica tra convergenze e divergenze che caratterizzano questo corpus di testi, lasciando intravedere ora repliche fedeli ora rielaborazioni locali di temi ricorrenti anche altrove. Per esempio, il riferimento a Mnemosyne, in cui è stato visto un diretto richiamo alle dottrine pitagoriche ${ }^{40}$ (non certo sorprendente per l'area crotoniate) così come la definizione di "figlio di cielo stellato" che l'iniziato presenta come 'password' al suo ingresso nell'aldilà, sono condivisi dalla lamina di Hipponion, oltre che da quelle della Tessaglia e di Creta.

Invece, una variante locale riguarda la definizione dello status acquisito dall'iniziato dopo la morte, che nella lamina di Petelia viene qualificato come 'eroe', mentre a Hipponion si fa riferimento alla sua appartenenza ai mus ta i $\mathrm{k}$ a i fba $\mathrm{k} \times \mathrm{o}$ i e a Thurii al divenire 'dio anziché mortale (qeof d"e hi a ht i fb r ot oi $\theta$ ). Queste variazioni sono probabilmente imputabili al «divario tra adepti a esperienze misteriche diverse $\gg^{41} \mathrm{o}$ a differenze tra comunità religiose pur legate da una comune fede escatologica. Su questo punto, pertanto, si pone l'opportunità di verificare «se o come contesti culturalmente e territorialmente diversi possano aver recepito o modificato un modello religioso comune». ${ }^{42}$

Nell'ambito della Magna Grecia la lamina di Petelia presenta più spiccate convergenze tematiche e formulari con quella di Hipponion che con quelle della vicina Thurii, ove l'attestarsi di più lamine ripartite tra diversi contesti sepolcrali lascia intravedere forme di 'personalizzazione' e di adattamento formulare ai diversi ambienti sociali di rispettiva pertinenza. ${ }^{43}$

Di documenti di ambito funerario redatti in lingue diverse dal latino resta pochissimo. Non si ha nessuna epigrafe sepolcrale in lingue indigene, mentre di epitafi redatti in greco non si dispone di altro che di due testi già noti al Kaibel con la formula di commiato consueta in età ellenistica: $x$ a i fe $(\mathrm{nn} .6 \mathrm{a}, \mathrm{b})$. In questi due testi merita segnalare che l'unico nome integralmente leggibile è l'antroponimo greco Euodoj ( $\mathrm{n}^{\circ} \mathrm{6a}$ ): lo stesso tipo di composto si ritrova come cognomen

40 Cfr. Pugliese Carratelli 2001, p.53, ma cfr. anche Ghidini Tortorelli 2006, p. 37.

41 Citazione da Ghidini Tortorelli 2006, p. 37.

42 Citazione da Ghidini Tortorelli 2006, p. 37.

43 Cfr. Musti 2005, p. 197. 
femminile nell'epitafio di una donna di età imperiale: Eubodis $\left(\mathrm{n}^{\circ} 13\right)$.

La continuità dell'antroponimia greca con il trasferimento di modelli morfologici al latino è manifestata dal più rappresentativo numero di epigrafi sepolcrali disponibili per l'età romana. Anche se non si hanno documenti in latino antecedenti l'età imperiale, certamente il contatto con Roma deve essersi attivato già molto prima, non solo in conseguenza della riduzione della vicina Crotone a colonia romana nel 193 a.C., ma soprattutto in conseguenza dell'atteggiamento filoromano della città in occasione della II guerra Punica.

Cionondimeno fino ad epoca tardo-repubblicana il greco resta saldamente la lingua dominante nella vita della città, almeno per le classi elevate, collocandosi ai livelli comunicativi formali, come mostrano l'iscrizione dei ginnasiarchi $\left(\mathrm{n}^{\circ} 7\right)^{44}$ e quella della coppia di magistrati eponimi su bollo laterizio noto in più esemplari $\left(\mathrm{n}^{\circ} 10\right),{ }^{45}$ entrambe databili tra fine III e II secolo a.C. ${ }^{46}$ I due documenti riflettono modelli istituzionali e tipologie testuali greche: da una parte, l'istituzione di ginnasi e la relativa carica di ginnasiarchi, che, come dichiara l'iscrizione, si incarica di compiti amministrativi, quali il rifacimento di un portico; dall'altra, l'attività di un'officina di laterizi, la cui natura pubblica è contrassegnata dalla sigla dh (mo \$ i on ) che accompagna, secondo l'abitudine greca, la menzione dell'organismo statale. La ginnasiarchia inserisce pienamente Petelia nelle strutture istituzionali delle città greche: i modelli educativi rappresentati dai 'ginnasi' e le competenze amministrative e finanziarie demandate alla carica dei ginnasiarchi, ben documentate dall'epigrafia delle città siceliote. ${ }^{47}$ Invece, più difficilmente definibile e marginale in questa sede è la questione se la magistratura diarchica rappresentata dai bolli su mattone sia identificabile con la carica dei ginnasiarchi, ai quali, se così fosse, si dovrebbe conferire una funzione eponima. ${ }^{48}$

44 IG XIV 637; Intrieri-Zumbo 1995, Costabile 2007a, p. 369.

45 Intrieri-Zumbo 1995, p.269 B-14; Costabile 2007a, p.365.

46 Cfr. Cordiano 1997, p. 64; Costabile 2007a, p.368-370.

47 Per il dossier documentario cfr. Cordiano 1997.

48 Ci limitiamo qui a segnalare l'ipotesi di Gasperini 1986, p.149 ss., secondo il quale il bollo laterizio recante i due magistrati eponimi e la sigla dh (mo \& i on) sarebbe riferibile ai duoviri municipali della vicina Crotone. In tal caso resta, tuttavia, sorprendente non solo l'uso del greco, ma anche di una tipologia testuale greca per la magistratura di una colonia romana. 
In ogni modo quanto preme sottolineare è che l'onomastica di questi documenti è di chiara matrice italica, e segnatamente appartenente all'antroponima osca. Il radicamento dell'onomastica osca in un contesto ellenofono emerge anche da altro genere di iscrizioni (cronologicamente omogenee) che menzionano 'Petelini all'estero', a testimonianza delle relazioni e dei contatti che la città intratteneva in ambito mediterraneo di età tardo-ellenistica. Un individuo di dichiarata provenienza da Petelia figura in più iscrizioni a Delo $\left(\mathrm{n}^{\circ} 8\right)$, mentre un altro si trova nell'elenco dei thearodokoi da Delfi $\left(\mathrm{n}^{\circ} 9\right)$.

Questi testi, però, mostrano diverse tipologie di adattamento e di combinazione delle denominazioni personali anelleniche offrendo una significativa campionatura:

a) designazione mediante il solo prenome in funzione di idionimo (rispettivamente L eukioj e N ouloj ) seguito da una sigla nella coppia di magistrati eponimi epifL eukibu or kaifNouibu El e $\left(n^{\circ} 10\right)$. Le sigle possono essere riconducibili ai rispettivi gentilizi, ma la loro condizione conferisce piuttosto l'aspetto di demotici segnalando l'omologazione, almeno a livello ufficiale, al sistema delle designazioni personali greche;

b) formula onomastica completa nel caso dell'iscrizione dei ginnasiarchi (M ihatoj Kriftioj M inafou [M eni]dajo[M ati]laje

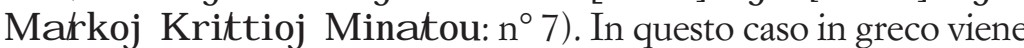
trasposta al completo la formula onomastica binomia di tipo italico. I due individui sono probabilmente fratelli ${ }^{49}$, uno dei quali continua il prenome paterno ed è provvisto di un cognomen grecanico.

c) designazione mediante il solo gentilizio in funzione di idionimo ('O $\mathrm{f}$ a / I i oj ) nella lista dei thearokodoi a Delfi $\left(\mathrm{n}^{\circ} 9\right)$.

d) idionimo greco e nome paterno osco nel personaggio di Delo 'Aga law $n$ N umy ibu ( $\left.n^{\circ} 8\right)$. Esempi di un idionimo greco a fronte di un patronimico osco sono numerosi ad Entella (dove non si ha l'opposto $)^{50}$ e altrove, come nel caso di AI kipoj M a Itkou nella lista di thearodokoi di Epidauro. ${ }^{51}$

Le quattro tipologie designative rispecchiano i diversi effetti del

49 Cfr. Cordiano 1997, p. 64.

50 Come nota Lejeune 1982, p. 793.

51 Cfr. De Sanctis 1911, p.445; Manganaro 1964, p.418; Poccetti 1989, p. 118 
contatto tra il sistema onomastico greco e quello osco con duttilità di combinazioni che spiccano in situazioni 'sinfasiche' di livelli sociolinguisticamente 'alti'. In particolare il confronto tra le due formule eponimiche (rispettivamente nn. 7,10) mette in rilievo due diversi processi di adattamento ai modelli greci che riguarda non solo l'onomastica, ma anche le strategie sintattiche della coordinazione delle rispettive coppie di nomi. La formula dei magistrati eponimi nei bolli su mattone è più aderente ai modelli greci rispetto a quella dell'iscrizione dei ginnasiarchi: la prima si costituisce dell'idionimo e di sigle in funzione di demotici (qualunque ne sia l'origine); nella seconda, invece, le designazioni rispecchiano la canonica struttura trimembre di prenome+gentilizio+patronimico delle denominazioni ufficiali italiche.

Inoltre, l'aggiunta di un grecanico (letto ora M eniddaj o

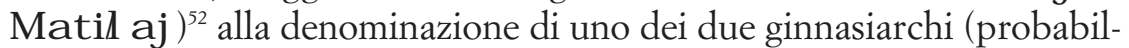
mente fratelli, a giudicare dall'omonimia del prenome paterno tipicamente osco, M i ha toj ), ne lascia intravedere l'adozione di un cognomen, pratica nota anche all'antroponimia osca. ${ }^{53}$ L'altro ginnasiarca porta, invece, un praenomen ( $\mathrm{M}$ a $\nmid \mathrm{k}$ oj $)$ che sembra essere l'adeguamento di Mamerkos osco. Il conguaglio di Mamerkos con M a f $\mathrm{k}$ oj non implica necessariamente un influsso romano o comunque un filtro latino, essendo noto anche in altre circostanze e in epoche antecedenti: per esempio, nel caso sopra citato di AI kipoj M a fkou menzionato nella lista di thearodokoi di Epidauro datata alla metà del IV secolo e, per questo, ritenuto figlio di Mamerkos tiranno di Catania messo a morte da Timoleonte. ${ }^{54}$ Altro caso di oscillazione tra Mamerk- e Marknelle fonti greche senza necessariamente un filtro latino riguarda la duplice tradizione del toponimo campano di orgine etrusca M a ma rkina / M a rkina. ${ }^{55}$

Le formule eponimiche dei testi nn. 7 e 10 si distinguono anche per le strutture coordinanti: la coppia di nomi sul bollo laterizio è unita dalla particella ka i/, quella dei ginnasiarchi dall'asindeto. Delle due

52 La lezione M a till a j proposta da Costabile 2007a, , corregge la precedente M enida j accolta in Costabile 1984.

53 Cfr. Lejeune 1976, p. 50 s.

54 Cfr. Manganaro 1964, p. 418. Più cauto su questa possibilità di identificazione Tagliamonte 1994, p. 146.

55 Cfr. Poccetti 1989, p. 118 ss.. 
strutture sintattiche la prima è più comune in greco, ${ }^{56}$ la seconda, invece, è più diffusa in ambiente sabellico e romano. ${ }^{57}$ Questo diverso comportamento sintattico risalta anche nelle iscrizioni bilingui greco-latine ove si menzionano coppie di fratelli ${ }^{58}$ come quella da Delo: L(ucius) L(ucius) Orbieis L.l(iberti) L eukioj Đrbioj L eukibu Likinoj ka if Leukioj Drbioj Leukibu Difiloj.

Questo particolare converge con i comportamenti onomastici dei rispettivi testi nell'additare diversi effetti del contatto tra sistemi linguistici. Infatti, nelle strutture tanto delle designazioni personali quanto della coordinazione, la formula del bollo laterizio riproduce più fedelmente il modello greco, mentre l'iscrizione dei ginnasiarchi traspone in greco non solo strutture di designazione personale, ma anche il legame sintattico di tipo italico. L'iscrizione dei ginnasiarchi, pertanto, manifesta la persistenza delle formule onomastiche e delle strutture sintattiche dell'osco in contesti ufficiali, che vengono, invece, obliterate in altri documenti. Quest'ultimo caso potrebbe definirsi, parafrasando una celebre espressione di Rohlfs, come spirito italico in veste greca.

La commistione tra le componenti linguistico-culturali italiche e quelle greche ha riflessi significativi anche in età romana, di cui l'onomastica costituisce interessante specchio. Continuatori di antichi nomi individuali greci sono cognomina (come Aphrodisius, Aphrodisia, il già citato Euhodis) $)^{59}$ e nuovi gentilizi creati su basi greche come Batbilius che presuppone un nome individuale locale B a qul (l )oj ( $\left.{ }^{\circ} 12\right)$. Altri nomi sono il risultato dell'interferenza morfologica tra i due sistemi come Crotonis che occorre come cognomen femminile (Octaviae Crotonidi) ${ }^{60}$ consistente in un derivato dal nome della vicina Crotone mediante il suffisso greco - ij / - i doj . Tale formazione, legata alla produttività di questo elemento morfologico, anche come marca di femmi-

56 Es. nei decreti di Entella: ep i fa ix oht wn A)rtemidw to u to u = i el ou ka if Gnaibu Oppibu (Dubois 1989, nn. 208, 211); nel decreto da Araxa: epifierewn $y$ Q rzagotou ka ifM hnokritou (Pouilloux 1960, n4).

57 Come mostrano le formule di magistrati in diarchia del tipo latino $\mathrm{Cn}$. Pompeio, M. Crasso consulibus (Caes. Gall. 4,1,1), dell'osco M.Siuttiis M. N.Púntiis aídilis (Rix 2002, Po 1) o dell'umbro maronatei Uois.Ner Propartie T.U.Uoisiener (Rix 2002, Um 10)

58 CIL I ${ }^{2}$ 2252= ILLRP 755.

59 CIL X 8087; AÉp. 84, 272.

60 Cfr. Gasperini 1986, p.153-154. 
nile (es. Turrhnol : Turrhni \}; E|| hn: E| | hni | ) ${ }^{61}$ si affianca ad altri etnonimi derivati dal nome della città: Krotwniathj / K rotwniati i, K rotwhioj / K rotwnia, K rotwniata. ${ }^{62}$

Ancor più interessante è la distribuzione funzione funzionale-contestuale del nome individuale $\mathrm{K}$ el a doj / Celadion in un epitafio bilingue, relativo ad un bambino morto a cinque anni. L'epigrafe sepolcrale è stata eretta dalla 'troupe' di un pantomimo, di nome Ionico (grex Ionici pantomimi), probabilmente padre del piccolo, che veniva forse utilizzato nelle attività sceniche $\left(\mathrm{n}^{\circ} 11\right){ }^{63}$ L'iscrizione si compone di un'intestazione in latino, che enuncia il curatore della sepoltura con i dati anagrafici del defunto, e di un epigramma in distici in greco. Nella parte poetica figura $\mathrm{K}$ el a doj, suo nome ufficiale, nella parte latina si trova Celadion, derivato con il suffisso ipocoristico -ion. Questo suffisso, comune nell'onomastica greca, ha contaminato anche quella latina della regione, come mostra il nome di A I fioj P rimi wn nella tegola di Pellaro, dove -ion si è sovrapposto a -iõn ${ }^{64}$ Celadion e $\mathrm{P}$ r i mi w $\mathrm{n}$ convergono nel rivelare la diffusione dell'ipocoristico greco in latino in modo inverso e speculare: nel primo caso (Celadion) si applica ad un nome greco trasferito in latino, nel secondo ( $\mathrm{P}$ r i mi $\mathrm{W} \mathrm{n}$ ) ad un nome latino trasferito in greco. Inoltre, la presenza della forma ipocoristica greca nel testo latino, che contiene il riferimento al nome del padre e all'ambiente professionale in cui il bambino è stato cresciuto, segnala che l'appellativo, con il quale il piccolo era identificato nel mondo dei suoi affetti, era greco.

\section{LA NUOVA TABELLA DEFIXIONIS}

Questo quadro documentario del plurilinguismo di Petelia si è recentemente arricchito di una nuova importante iscrizione su lamina di piombo, appartenente alla categoria dei testi maledizione, pubblicata e commentata da M.L.Lazzarini. ${ }^{65}$ Il testo non incrementa solo numericamente il dossier epigrafico, ma ha anche una straordinaria potenzialità documentaria in relazione ai fenomeni di interferenza tra osco e greco.

\footnotetext{
61 Cfr. Chantraine 1933, p. 342 ss.

62 Elencati da Stefano di Bisanzio s.v. K r otw n.

63 Ci riferiamo alla convincente interpretazione di Lazzarini 2004b.

64 Cfr. Lazzarini-Lattanzi-Mosino 1979.

65 Lazzarini 2004a; 2009; 2012.
} 
Innanzitutto il nuovo testo accresce anche il corpus delle tabellae defixionis osche, sottolineando la particolare concentrazione di questi testi nell'area lucano-bruzia, che non solo supera numericamente, ma anche antecede cronologicamente quelle di ambiente campano-sannita. A tale specifica arealità soggiacciono almeno tre ordini di ragionii: a) la forte compenetrazione della cultura greca, da cui questo tipo di testualità si è irradiata in Occidente; b) l'articolazione sociale e politica di tipo urbano o paraurbano; c) la condizione di particolare diglossia che favorisce l'emergere dell'osco in questo tipo di testi, in quanto codice che nel repertorio sociolinguistico viene riservato a livelli comunicativi informali o di minor prestigio.

Nella nuova tabella defixionis da Petelia, che è tra le più lunghe e complesse che coinvolgono l'osco la scrittura si dispone su quattro colonne in cui è sezionata la superficie della striscia di piombo. Tre di esse non contengono che una lista di nomi disposti in coppia per ciascuna linea. La quarta colonna, tranne forse la prima linea, è sostanzialmente riservata alla formula di imprecazione in cui si mescolano greco e osco.

In ciascuna delle coppie di nomi elencate in colonna sono riconoscibili formule onomastiche binomie di tipo italico riconducibili alle seguenti tipologie:

a) Nomi di repertorio osco con morfologia osca in nominativo:

c. I : П $\alpha \kappa о \lambda \Sigma \tau \alpha \tau \iota \varepsilon \varsigma$ (1.3); $M \alpha \rho \alpha(\varsigma) \Sigma \tau \alpha \tau \iota \varepsilon \varsigma(1.4) ;$

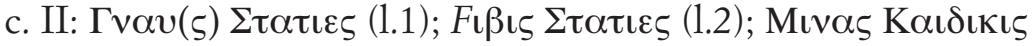
(1.6);

c. III: $\mathrm{A} F \varepsilon \varsigma \mathrm{A} v \delta \alpha \iota \varsigma$ (1.1).

b) Nomi di repertorio osco con morfologia non osca:

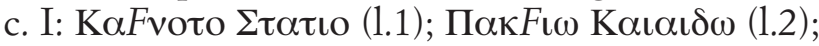

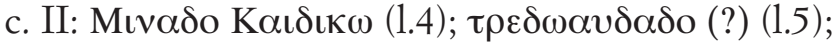

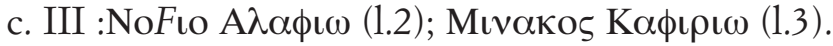

c) Accoppiamento di nomi greci e antroponimi oschi con morfologia non osca:

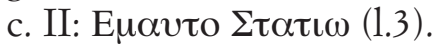

Occorre innanzitutto constatare la scrupolosa osservanza del sistema binomio in tutte le designazioni personali che occupano le prime tre colonne del testo. Come abbiamo visto sopra, tale condizione si presenta nel dossier epigrafico solo nell'iscrizione dei ginnasiarchi. Il diverso statuto sociolinguistico di questi due documenti (l'uno informale e privato, l'altro ufficiale e pubblico) e il loro divario cronologico 
convergono nella conclusione che il sistema gentilizio di impronta italica ha avuto un radicamento diastratico ed una sua continuità diacronica nella vita della città pur nella profonda ellenizzazione. Le diverse strategie designative, in cui viene selezionato ora l'uno ora l'altro dei due elementi, secondo la campionatura sopra schematizzata sulla base dei testi (nn.7-10) sono legate alle scelte personali o a specifici contesti situazionali. Anche l'adozione di un antroponimo greco non implica necessariamente l'abbandono del sistema e del repertorio indigeno, a cui potrebbe far pensare il caso del petelino emigrato a Delo 'A ga q $q \mathrm{w} n$

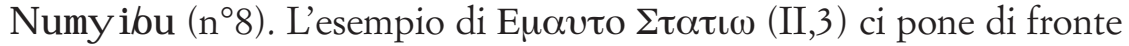
ad un individuo della gens Statia, che ha sì adottato un idionimo greco ${ }^{66}$ come prenome, ma ha conservato la designazione binomia con il gentilizio. Tra l'altro, questa gens annovera un numero elevato di esponenti nella tabella defixionis in questione: almeno la metà delle designazioni personali binomie chiaramente riconoscibili $(6: 12)$ appartengono agli Statii. Solo 1 su 6 presenta un nome greco come nome individuale, che è segnale di una certa resistenza dell'onomastica indigena che, almeno tra i petelini sembra infranta solo dal caso dell'emigrato a Delo 'A ga quw n N umy ibu.

Invece, possibile origine greca potrebbe avere il gentilizio Kaфı$\rho \iota \omega$, se è tratto da $\mathrm{Kafhr} \mathrm{ei}^{\mathrm{j}} \mathrm{j}$, aggettivo derivato dal toponimo dell'Eubea K a f hreul. Gentilizi oschi derivati dal lessico o dall'onomastica del greco non sono rari (es. Pítakiis < P i ga koj; Smintiis < S minqeul, attributo di Apollo o $S$ mihgh toponimo micrasiatico; $\Sigma v \rho \iota \varepsilon \varsigma<S$ u $F_{0 j}$ ). Tuttavia, è anche possibile un'origine italica, a cui rimanderebbero i gentilizi latini Cafrius e Cafranius, oltre a Cafre e Cafrial in etrusco, le cui attestazioni rimandano all'Italia centro-setten-

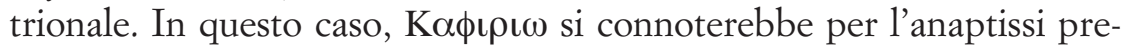
sente anche in $\mathrm{A} \lambda \alpha \phi \omega \omega$.

Altro nomen che vanta più di una occorrenza nel testo della

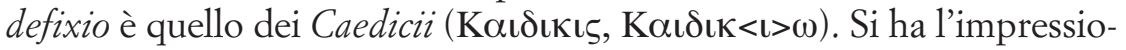
ne che bersaglio della maledizione sia un intero clan gentilizio locale nelle cui azioni, che motivano l'imprecazione, sono coinvolti esponenti di qualche altra gens locale. Questa tipologia rientra nella prassi comune alle tabellae defixionis, in cui si associano persone legate da vincoli

66 Per i riferimenti alla diffusione di 'E ma ut ol' come antroponimo anche in Magna Grecia, cfr. Lazzarini 2009, p.429. 
familiari insieme ad altri che si rendono complici o corresponsabili dell'oggetto di esecrazione. Nei testi greci si indicano spesso le relazioni familiari attraverso le specifiche di termini di parentela ('moglie, figlio, fratello/sorella' $)^{67}$ o con formule omnicomprensive del tipo tou f $f$ met a $f$ PI atahhj pahtaj kaifahdraj kaifgunaikaj; ${ }^{68}$ oi (metaftout

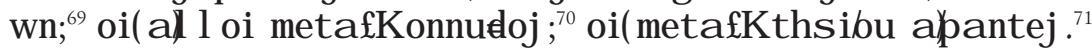

Nelle defixiones osche e latine i legami parentali vengono più spesso segnalati dalla condivisione del gentilizio. Così, per esempio, in una defixio di Capua due personaggi che aprono la lista dei 'defissi' condividono il gentilizio Vírriis, ${ }^{72}$, in una defixio di Cuma pur estremamente frammentaria, spicca la ripetuta menzione del gentilizio Rabiis. ${ }^{73}$ Allo stesso modo, ricorsività onomastiche di gentilizi si colgono nella complessa serie di designazioni personali che compongono la defixio

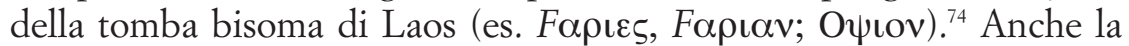
defixio latina da Delo (unico documento latino non lapidario dall'isola egea nella sua fase di 'porto franco', che rispecchia un ambiente culturalmente misto romano-italico) reca segni di complesse associazioni familiari. ${ }^{75}$ Qui due coppie di fratelli (A.Q. Paconios M.f. e N.M.Raios) che si inseriscono in un dissidio familiare che divide per ragioni ignote la gens Paconia. Infatti tra i suoi componenti (ovviamente presenti sull'isola) figurano sia alcuni defissi (in testa L. Paconium senem, insieme a A.Q. Paconios M.f. e Cn. Paconius Apollonius, elencati successivamente) sia il defiggente, che si nomina nella formula riassuntiva finale (et si quis alius erit inimeicus Tito Paconio).

Dal testo di Petelia si riceve l'impressione che l'azione che motiva l'iniziativa di maledizione converge su un gruppo consistente di individui appartenenti allo stesso clan gentilizio, intorno ai quali ruotano altri perpaidion.

76 Si veda l'indice di Wünsch 1897, p. 48 s.vv. a blel fol, a del f h, gu nh /p a i F,

68 Audollent 1904, n 61.

69 Wünsch 1897, n 57, 17.

70 Wünsch 1897, n $57,9$.

71 Wünsch 1897, n 101,9.

72 Rix 2002, Cp 36.

73 Rix 2002, Cm 14.

74 Rix 2002, Lu 46.

75 ILLRP 1150 = CIL I2 3439. Rilettura e importante commento del testo sono stati fatti da Solin 1982. 
sonaggi. Il testo non permette di stabilire quale sia il criterio della loro distribuzione nelle liste che occupano le singole colonne. E' probabile che la loro ripartizione sia dettata dal livello di coinvolgimento personale nell'azione che ha motivato l'atto di imprecazione. Possiamo solo constatare che i componenti della gens Statia si addensano nelle prime due colonne. Nella seconda colonna che ospita il numero più alto di individui rispetto alle altre si concentrano ben tre Statii e due Caedicii, che costituiscono i due gentilizi più rappresentati nel testo. La terza colonna elenca tre individui tra loro eterogenei concludendosi con l'indicazione di due etnonimi legati dall'asindeto (B $\alpha v \tau \iota v \omega \mathrm{K} \omega \sigma \sigma \alpha v \omega)$. Tale specifica onomastica, insieme alla loro collocazione alla fine della lista, marca i nomi personali di riferimento rispetto a tutti gli altri che non sono accompagnati da etnonimi. Con ogni probabilità, l'indicazione degli etnonimi sarà da ascrivere ad individui che si trovano nella condizione di 'stranieri' o di cittadinanza non pienamente acquisita. Non è il caso di entrare qui nella ridda di ipotesi possibili che discendono dall'attribuzione dei due etnonimi ad uno stesso individuo o a più individui. Ci limitiamo al rilievo di alcuni dati sicuri che discendono dalla loro menzione in quel contesto:

a) la mobilità personale tra gli Italici del sud al volgere del IV secolo che si inserisce in un quadro più ampio di pluralismo 'multietnico' degli insediamenti costieri di ambito bruzio e lucano, segnalato anche dall'onomastica personale di altre defixiones, come quelle di Laos e di Roccagoriosa. ${ }^{76}$

b) La permeabilità specifica di Petelia all'afflusso di elementi di altre comunità sia del Bruzio sia della Lucania. Al Bruzio è, infatti, probabilmente ascrivibile K $\omega \sigma \sigma \alpha v \omega$ se tale nome è in rapporto con la K os S a citata da Ecateo come pol ij 0 inwtrwa eh mes 0 -

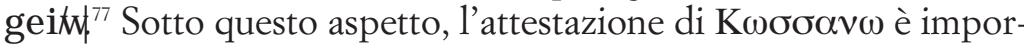
tante perché documenta la continuità insediativa del sito almeno fino alla fine del IV secolo con importanti ricadute di ordine storico-topografico tra fase sannita e pre-sannita. ${ }^{78}$

76 Rix 2002, Lu 45, Lu 63.

77 Hec. Frg. 77 Nenci. Ulteriori riferimenti al frammento di Ecateo in Lazzarini 2009, p.429. Per questioni di topografia storica relative al toponimo ecataico e per altre sue possibili fonti cfr. Zancani Montuoro 1976.

78 Sulla continuità discontinuità insediativa dei siti 'enotri menzionati da Ecateo e questioni connesse cfr. Lombardo 2001. 


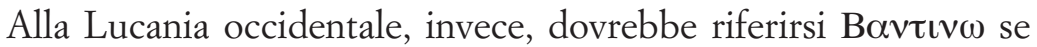
tale etnonimo è da identificarsi con il noto sito di Bantia da cui proviene la lex Osca e non con un centro omonimo altrimenti ignoto. E' evidente che l'attestazione del nome di Bantia oltre due secoli prima della sua menzione nella nota lex Osca ha le sue dovute implicazioni sull'importanza del sito anche in diacronia.

c) La distinzione tra cittadini e 'stranieri' inseriti nella vita della città con probabili connotati giuridici diversi rende lecito domandarsi in che misura Petelia condividesse il modello delle città greche per quanto attiene i livelli di integrazione degli 'stranieri' relativamente al godimento dei diritti civili e politici.

Una delle questioni nodali del testo riguarda la relazione sintattica tra due diverse categorie morfologiche in cui si ripartiscono i nomi presenti nell'iscrizione sulla lamina plumbea. Da una parte, infatti, si hanno gruppi di nomi in nominativo singolare osco, con relativa distinzione

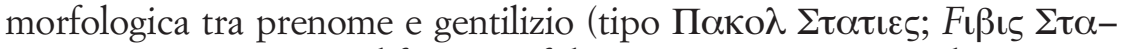

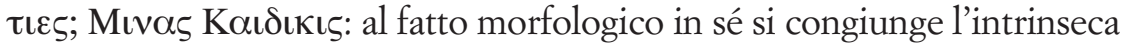
specificità dell'osco per cui «il morfema $-y o$ - assume aspetto diverso a seconda che si trovi nel primo o nel secondo elemento della formula binomia». ${ }^{79}$ Dall'altra, invece, gruppi di nomi che si sottraggono all'im-

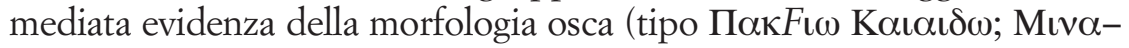

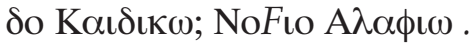

Per questi ultimi in linea di principio possono scalarsi due ipotesi di lettura, che comportano due diverse strutture sintattiche, l'una in osco, l'altra in greco.

La chiave di lettura in osco, puramente speculativa, non può che prendere in considerazione lo statuto di accusativi singolari. Tale circostanza permetterebbe un apparente parallelismo testuale con le strutture sintattiche di altre defixiones di ambiente bruzio-lucano marcate dall'opposizione tra nominativo e accusativo (es. Т $\rho \varepsilon \beta \alpha \varsigma$ Т $\rho \varepsilon \beta \alpha \tau \iota \varepsilon \varsigma$

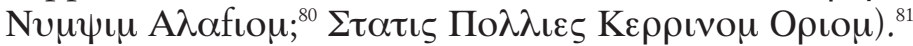

Tuttavia, l'identificazione di morfi di accusativo singolare dell'osco non regge alla prova dei fatti per le seguenti ragioni: 1) non si realizza il distinto trattamento morfologico atteso tra prenome gentilizio

\footnotetext{
79 Citazione da Prosdocimi 2004, p. 1476.

80 Rix 2002, Lu 43.

81 Rix 2002, Lu 44.
} 


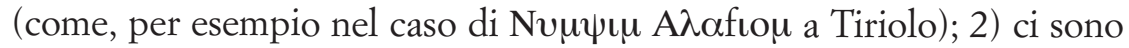
forme inspiegabili con la marca di accusativi (es. Mıvokos); 3) la distribuzione dei presunti accusativi rispetto ai nominativi non è isomorfa rispetto a quella delle altre defixiones bruzio-lucane, dove un nome in accusativo si giustappone ad uno in caso nominativo.

Il modello flessionale greco ammette due alternative possibili: a) nominativo singolare, caso che consente l'omogeneità sintattica con il caso costantemente seguito nei nomi con flessione osca; b) genitivo singolare con vocalismo di tipo 'dorico' indicato dall'uso incoerente dei grafi $\langle 0\rangle$ e $\langle W\rangle$. La patina dorica dell'iscrizione è segnalata dal vocativo $\mathrm{H}$ er ma $=\mathrm{e}$ ben si inserisce nei connotati dialettali dell'area crotoniate a cui appartiene Petelia. ${ }^{82}$

A sfavore del nominativo giocano due controindicazioni, una di natura grafica ed una di ordine linguistico. L'anomalia grafica consiste

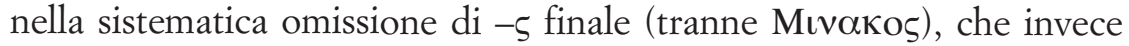
viene costantemente notata nella flessione osca, salvo quando la stessa consonante si presenta nell'iniziale della parola successiva, come si veri-

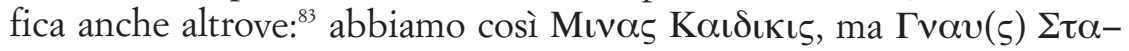
$\tau \iota \varepsilon \varsigma ; M \alpha \rho \alpha(\varsigma) \Sigma \tau \alpha \tau \iota \varepsilon \zeta ; F \iota \beta \iota(\varsigma) \Sigma \tau \alpha \tau \iota \varepsilon \zeta$. L'anomalia linguistica è connessa alla spiegazione come nominativi, in quanto comporta la separazione dei nomi Mıvado e Mıvakos dal prenome osco Mıvas < Minatøs, ricorrente più volte nel testo.

Invece, l'interpretazione delle forme non osche con il caso genitivo ha il vantaggio, come abbiamo mostrato altrove, ${ }^{84}$ di poter giustifica-

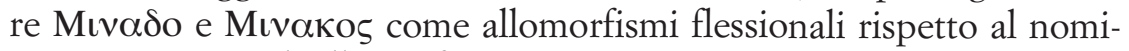
nativo Mıvas. Tali allomorfismi poggiano su un fenomeno ben noto in livelli non sorvegliati del greco, per cui un nominativo uscente in $-\alpha \varsigma$ poteva seguire diversi modelli della flessione e cioè - $-\varsigma_{\varsigma} /-\alpha v \tau o \zeta ;-\alpha \varsigma$ /

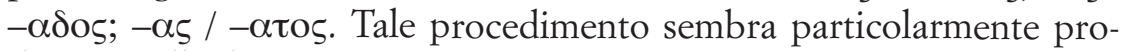
duttivo nell'adattamento di nomi alloglotti alla flessione greca, come si verifica già nella ricezione di nomi stranieri nell'Atene di $\mathrm{V}$ secolo. ${ }^{85}$ Soprattutto l'onomastica si fa modello e veicolo, come anche in altre

82 Cfr. Lazzarini 2003b.

83 Per esempio nella defixio osca da Laos (Rix 2002, Lu 63) con incostanza: es.

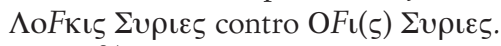

84 Cfr. Poccetti 2006.

85 Cfr. Cassio 1985. 
circostanze, di mutamento morfologico anche per il lessico, sfruttando tendenze latenti nel sistema. ${ }^{86}$

In concreto, dunque, l'analisi di Mıvado e Mıvakos come genitivi, allomorfi nella flessione di un tema uscente in - $\alpha \varsigma$ al nominativo, indifferentemente come tema in - $\alpha$ K o come tema in $-v \tau-/-\delta$ - (questi ultimi divenuti già omofoni nell'antichità) accredita la lettura come genitivi dei nomi con morfologia non osca.

Se così è, sembra profilarsi una ripartizione che pare rigorosamente perseguita nelle prime tre colonne del testo tra il nominativo espresso sempre in osco e il genitivo espresso sempre in greco. La sola eccezione potrebbe realizzarsi all'inizio dell'ultima colonna, se nella sequenza $\pi \alpha F \varepsilon \lambda \iota s$ è da identificare un antroponimo in nominativo singolare del greco standard o in nominativo plurale osco (tipo $\Delta \iota \rho \iota s$ nell'iscrizione da Atena Lucana). ${ }^{87}$ Tuttavia l'agnizione di un antroponimo è inficiata dalla difficoltà di identificazione del nome, se non a prezzo dell'isolamento di una sigla (insolita nel testo), dietro la scansio-

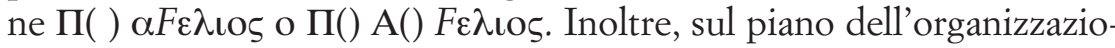
ne testuale, non si può non tener conto che la struttura della lista degli antroponimi si chiude con la colonna 3 e che la colonna 4 sembra destinata ad ospitare enunciati di altro tipo.

In conclusione, qualunque soluzione morfologica si voglia adottare per i nomi che non hanno palese morfologia osca restano aperti almeno due interrogativi : a) la ragione della distribuzione testuale tra $i$ nomi in caso nominativo (sempre osco) e quelli in caso diverso dal nominativo (molto probabilmente sempre in greco); b) la ragione della ripartizione, certo non casuale, tra le due lingue in rapporto alla flessione dei nomi stessi.

Un particolare ci sembra degno di nota. Tra gli esponenti della gens Statia gli unici individui la cui designazione personale non si pre-

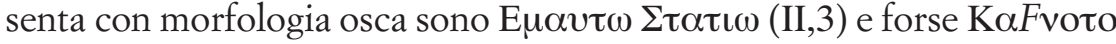
$\Sigma \tau \alpha \tau \iota \mathrm{I}(\mathrm{I}, 1)$. Non è forse casuale la circostanza che la flessione greca si accompagni alla presenza di due prenomi non appartenenti al repertorio osco: lo è sicuramente E $\mu \alpha v \tau \omega$ (che gode di puntuali riferimenti

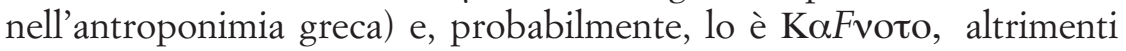

86 Sull'onomastica come modello di mutamento morfologico e tramite dell'induzione di morfemi cfr. Lazzeroni 1994.

87 Rix 2002, Lu 2. 
ignoto e, comunque, di non chiara pertinentizzazione. È, altresì, possibile che il nome sia allogeno, adattato alla morfologia greca. Tuttavia, la lettura, sulla base della foto e dell'apografo fornito, non appare del tutto nitida e necessita di un'ulteriore verifica.

In ogni caso, tale particolare, sia pure entro i limiti di una campionatura ristretta, suggerisce la conclusione che, mentre l'antroponimia osca viene morfologicamente grecizzata (es. ПакFı $\omega \alpha \iota \alpha \iota \delta \omega ;$

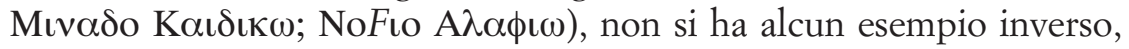
ovvero di 'oscizzazione' di antroponimi greci.

Per quanto riguarda le competenze linguistiche dell'estensore del testo, è opportuno far partire le riflessioni da alcuni fatti grafici. Il dato più saliente è la presenza di due segni per le vocali di timbro velare, trascritte rispettivamente con $<\mathrm{o}\rangle \mathrm{e}<\omega\rangle$ :

Tab. 1

Distribuzione dei grafi $<0>,<\omega>$

\begin{tabular}{|c|c|}
\hline 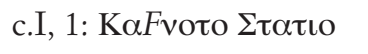 & 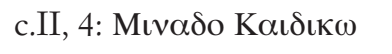 \\
\hline c. I, 2: П $\alpha \kappa F \iota \omega$ K $\alpha \iota \alpha \iota \delta \omega$ & 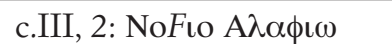 \\
\hline c.II, 3: E $\mu \alpha v \tau \omega \Sigma \tau \tau \alpha \tau \iota \omega$ & 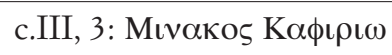 \\
\hline c. II, 5: $\tau \rho \varepsilon \delta \omega \alpha v \delta \alpha \delta o(?)$ & \\
\hline
\end{tabular}

I due grafi non presentano alcuna ripartizione funzionale tra prenome e gentilizio, contrariamente a quanto potrebbe evincersi dai casi

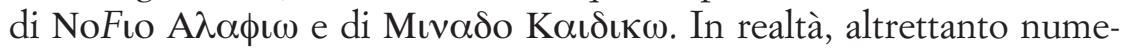
rosi sono i controesempi, dove tale differenziazione è annullata, in un

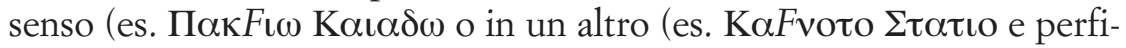
no invertita come in $\tau \rho \varepsilon \delta \omega \alpha v \delta \alpha \delta o$ (?) (se la sequenza è da suddividere $\tau \rho \varepsilon \delta \omega \alpha v \delta \alpha \delta o$ ). Gli antroponimi seguono la flessione dei temi in $-O$

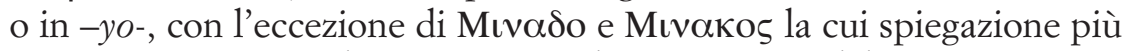
conveniente, come detto sopra, è il trattamento del prenome osco Mivas < Minatøs secondo il modello flessionale di temi in consonante divenuti allomorfi ( $-\alpha \kappa-,-v \tau-/-\delta-)$.

Tuttavia incoerenze nella distribuzione dei digrafi $\langle 0\rangle$ e $\langle\omega\rangle$ si constatano anche al di fuori dell'onomastica e colpiscono tanto l'osco quanto il greco.

Nella parte in osco un esempio di ipodifferenziazione tra i due grafi all'interno di una stessa parola è $\sigma 0 \lambda \lambda \mathrm{o} \mu$ se si tratta di genitivo plurale 
osco da *sollōm "di tutti". A sua volta la grafia $<0>$ della vocale morfe-

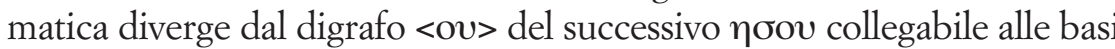
pronominali *eiso- o *ekso-. In principio, non ci sono ragioni fonetiche dirimenti per la scelta tra le due basi pronominali, anche se a favore di *eiso- milita la sua esclusiva funzione di anaforico, che meglio si adatta al testo (ed è usato in questo senso anche in altre defixiones osche) ${ }^{88}$ mentre ekso- figura più spesso in funzione di deittico. ${ }^{89}$

Per quanto riguarda il morfema desinenziale di $\eta \sigma o v$ il digrafo $<$ ou> difficilmente può rappresentare altro che l'esito di $/ \bar{o} /$, dal momento che si rendono possibili due soluzioni morfologiche: a) come genitivo plurale, $\eta \sigma o v(\mu)(<*$ eisōm o *eksōm) in concordanza sintatti-

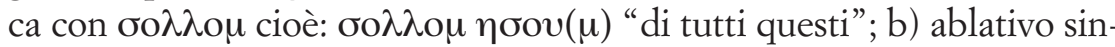
golare $\eta \sigma o v(\delta)$ (<*eisōd o *eksōd) con funzione locativale in parallelo alla determinazione locativale $\alpha v \tau e$ "=qui", con cui si chiude la formula in greco. Le due soluzioni comportano una diversa funzione del prenome, cioè anaforico nel caso a) e deittico, cioè legato al processo dell'enunciazione, nel caso b)

Comunque sia, sul versante del vocalismo si palesano incoerenze grafiche che toccano tanto l'uso di segni diversi per rappresentare esiti

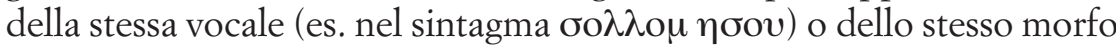
con identica funzione sintattica (i nomi della Tab. 1) quanto l'impiego dello stesso segno per indicare vocali di origine diversa, quali, per esempio, le vocali di $\sigma 0 \lambda \lambda \mathrm{o} \mu$.

Analogamente, nella porzione in greco si registrano la grafia

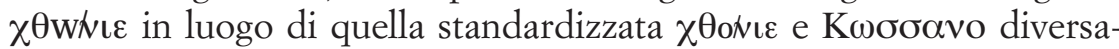

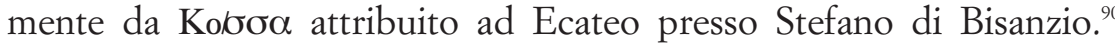
Pertanto, il quadro generale della distribuzione dei grafi $\langle 0\rangle$ e $\langle\omega\rangle$ sembra denunciare un'indifferenza nella disciplina dei due segni vocalici tanto nella trascrizione di nomi e elementi lessicali dell'osco quanto di nomi e di elementi lessicali del greco. Di conseguenza, si può desumere non solo l'assenza di norme e di coerenza ortografica nella scrittura dell'osco (che colpisce soprattutto la notazione delle vocali velari), ma anche una certa trascuratezza perfino nello scrivere il greco.

88 Cfr. eiseis : Rix 2002, Cp 37; eisunk : Rix 2002, Cp 37.

89 Cfr. Penney 2002, 132; Dupraz 2012, 169.

90 Sebbene quest'ultimo raffronto sia meno cogente per l'impossibilità di stabilire se l'originale di Ecateo prevdesse la distinzione grafica tra $\langle 0\rangle$ e $\langle\omega\rangle$. 
Altro fatto di inaccuratezza nella scripta greca riguarda le consonanti aspirate. Lasciando da parte dekeo in luogo di detxou che potrebbe anche imputarsi a selezione di varianti vocaliche interne al greco stesso, certamente non rispettosa dell'ortografia del greco è ка $\theta \varepsilon \kappa \varepsilon$ per $\kappa a ~ t \varepsilon \chi \varepsilon$ nella formula di chiusura del testo, dove è invertita la posizione delle aspirate.

Ci sembra che questi due ordini di negligenze convergano in un unico punto: il repertorio linguistico di chi ha redatto il testo era indifferente alle distinzioni fonologiche espresse sia dai grafi $\langle 0\rangle /\langle\omega\rangle$ sia dai segni per le aspirate. Tra l'altro, si ricorda che il segno per l'aspirata $\langle\phi\rangle$ era chiamato a notare la fricativa labiodentale italica /f/ tanto in $A \lambda \alpha \phi i \omega$

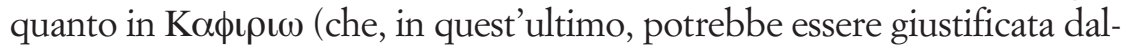
l'eventuale origine greca del nome, come detto sopra). L'uso di $\langle\phi\rangle$ in A $\lambda \alpha \phi \iota \omega$ distingue il nostro testo dalla defixio di Tiriolo dove lo stesso gentilizio $(\mathrm{A} \lambda \alpha f \mathrm{io \mu})^{91}$ reca uno dei segni consueti per rappresentare /f/ nella grafia osco-greca. Dunque anche nella notazione di $/ f /$ il documento mostra la non adesione al sistema grafico della scrittura osco-greca. Nello stesso tempo, l'adozione del segno per l'aspirata greca con il valore di fricativa da tempo converge con l'uso corrente in diverse varietà del greco. ${ }^{92}$

Riassumiamo nel seguente prospetto i principali fenomeni di variazioni e divergenze che si verificano, da una parte, in parole e nomi greci rispetto alla grafia 'standardizzata' del greco ellenistico e, dall'altra, nelle parole e nomi oschi rispetto alle codifiche della grafia osco-greca:

Tab. 2

\begin{tabular}{|c|c|c|}
\hline & Greco & Osco \\
\hline$<0>/<\omega>$ & 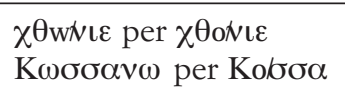 & $\begin{array}{l}\text { Cfr. Tab. 1; } \\
\sigma o \lambda \lambda \mathrm{o} \mu\left(<{ }^{*} \text { sollōm }\right)\end{array}$ \\
\hline$<\varepsilon>/<\eta>$ & & 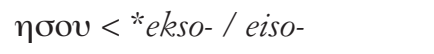 \\
\hline Consonanti aspirate & 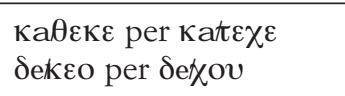 & $\begin{array}{l}\text { Uso di }<\phi>\text { con valore di }<\mathrm{f}> \\
\text { A } \lambda \alpha \phi \iota \omega ; \text { K } \alpha \phi \iota \iota \omega(?)\end{array}$ \\
\hline $\begin{array}{l}<F>\text { e }<v>\text { per la } \\
\text { semivocale } / w /\end{array}$ & E $\mu \alpha v \tau \omega$ & 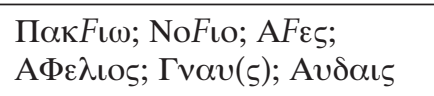 \\
\hline
\end{tabular}

91 Rix 2002, Lu 43.

92 Cfr. Arena 1983, 198 ss. 
Da notare, infine, un paio di grafie erronee, in un caso, per omissione di un segno e per dittografia, nell'altro:

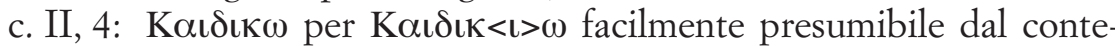
stuale Kaıঠıкıऽ (lat. Caedicius, ${ }^{93}$ osco Caedcies). ${ }^{94}$

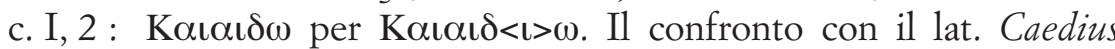
impone il riconoscimento della diplografia $\operatorname{Kal}\{\alpha \mathbf{l}\} \delta<\imath>\omega$. Tuttavia la presenza in peligno della forma Caiedia ${ }^{95}$ (se da Caiaidia) impone cautela in un tale emendamento.

L'insieme di questi fatti denuncia una sostanziale autonomia del testo di imprecazione tanto dall'ortografia greca quanto dalle norme dell'osco-greco. Rispetto all'osco-greco fatto saliente è l'uso del segno $\langle\phi\rangle$ per rappresentare la spirante labiodentale sorda in luogo delle molteplici varianti note nella scrittura osco-greca, di cui si ha anche una attestazione nel Bruzio (A $\lambda \alpha$ f in questo caso specifico l'adozione di $\langle\phi\rangle$ in luogo dei molteplici segni impiegati dall'osco-greco per rappresentare la fricativa labiodentale sia stata dettata dalla consapevole e più forte adesione al modello greco.

Per quanto riguarda la rappresentazione della semivocale /w/

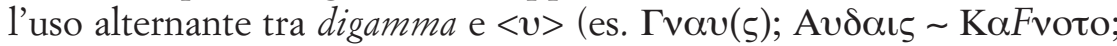
$\Pi \alpha \kappa F \iota \omega K \alpha \iota \alpha \iota \delta \omega)$ è forse imputabile all'interferenza tra il sistema grafico dell'osco e quello greco. Tuttavia, va anche ricordato che, per un verso, il segno del digamma si è attardato nelle scritture locali, come, per esempio, a Taranto e a Eraclea, ${ }^{97}$ e che, per un altro verso, relativamente alla notazione del digamma nei dittonghi la grafia dell'osco del Bruzio è assai meno rigorosa rispetto a quella della Lucania, ${ }^{98}$ ove tale consuetudine è esemplata sulla grafia osca campano-sannita. ${ }^{99}$ Pertanto anche in questa incoerenza il documento di Petelia potrebbe risentire tanto dell'assenza di una norma ortografica regionale nella scrittura dell'osco (legata anche alla minore forza di attrazione da parte del modello scrittorio campano-sannita) quanto delle variazioni presenti nell'epi-

93 Anche in contesto osco della Campania: Rix 2002, Cm 15; Mancini 1988.

94 Rix 2002, Lu 56.

95 Rix 2002, Pg 17.

96 Rix $2002 \mathrm{Lu} 43$.

97 Sull'uso del segno in area tarantina e nelle Tavole di Eraclea ancora in pieno IV secolo cfr. Moretti 1984, p. 497.

98 Cfr. Poccetti 1988, p. 153 ss.

99 Come mostrato da Lazzeroni 1983, p. 179. 
grafia greca coeva della Magna Grecia. Ma, a ben vedere, le due soluzioni non solo non si escludono tra loro, ma anzi si complimentano e forse si giustificano reciprocamente.

Passando ad aspetti più prettamente linguistici, la formula imprecatoria che sigilla la fine del testo è in greco. Apparentemente si tratta di un greco impeccabile, che, tuttavia, tradisce una certa trasandatezza grafica, propria di una non perfetta padronanza della lingua, proprio a livello della scrittura. La formula si compone, infatti, di varie espressioni ricorrenti con varia composizione in diverse defixiones greche e che, dunque, dovevano appartenere al repertorio comune degli operatori di magia. ${ }^{100}$

La formula greca è preceduta da una espressione in osco che presenta tratti altrettanto spiccati di inaccuratezza formale, come mostra l'incoerente notazione delle vocali velari, a cui abbiamo già fatto cenno

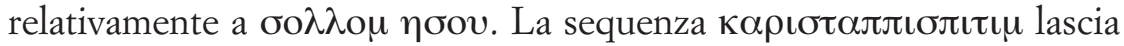
identificare una forma verbale seguita da un pronome relativo/indefinito $\iota \iota \sigma \pi \iota \tau$ spiegabile alla luce della glossa di Festo pitpit Osce quicquid. ${ }^{101}$ Per quanto precede $\pi \iota \sigma \pi \iota \tau$ la soluzione più probabile è un verbo al congiuntivo $\kappa \alpha \rho \iota \sigma \tau \alpha \delta$ (?) che sostanzia l'atto illocutorio dell'imprecazione. All'espressione, pertanto, è possibile attribuire un senso generico "sia inflitta una pena, una punizione a chiunque tra tutti questi" oppure "chiunque tra tutti (quanti nominati sopra) sia trascinato o punito qui”. Riflesso del parlato è probabilmente la consonante geminata $<\pi \pi>$ frutto di assimilazione consonantica o di rafforzamento fonotattico da $\kappa \alpha \rho \iota \sigma \tau \alpha \delta \pi \iota \sigma \pi \iota \tau$.

Di più ardua spiegazione è l'appendice $-\iota \mu$ in $\pi \iota \sigma \pi \iota \tau \iota \mu$. Se non si vuole ascrivere questa appendice sillabica a semplice effetto di assonanza per iterazione fonica della vocale precedente e della consonante finale della parola seguente (secondo un espediente non ignoto a que-

100 E' sufficiente uno sguardo all'indice di Wünsch 1897, p. 47-48 per constatare che nelle formule di maledizione dell'Attica Hermes è la divinità più invocata per attuare le maledizioni, così come l'attributo x qo hi oj è il più ricorrente. Altrettanta frequenza ha l'attributo $\mathrm{k}$ a $t$ ox oj collegato al verbo $\mathrm{k}$ a t ek w usato nella defixio di Petelia e che appare più sporadicamente nelle defixiones attiche. Altri riferimenti a Hermes e all'impiego 'tecnico' della famiglia lessicale ka toxoj e ka tekw si trovano in altre lamine plumbee ateniesi (cfr. Costabile 2007b). Invocazione a Hermes, ma con diverso formulario, appare anche nella defixio in dialetto dorico da Tiriolo (cfr. Lazzarini 1994).

101 P.-Fest. 235, 15. 
sto genere di testi), si può ipotizzare una grafia abbreviata o erronea per la particella di coordinazione inim, (da reintegrare $\langle\iota v\rangle(\mu)$, ${ }^{102}$ che potrebbe giustificarsi in due modi:

a) con vera e propria funzione coordinante rispetto alla frase contrasse-

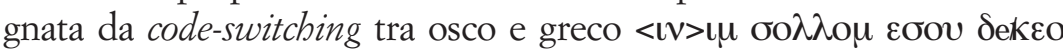

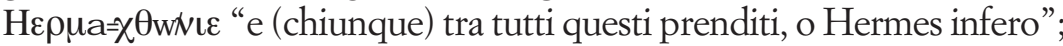

b) con uno dei valori contestuali paralleli a quelli assunti da et in latino e кaif in greco "anche, perfino, eppure". ${ }^{103}$ Il valore di 'anche' potrebbe qui vantaggiosamente applicarsi conferendo all'enunciato il senso di "sia punito anche (uno qualunque) tra tutti questi". In sostanza, il defiggente si augura come condizione sufficiente che l'effetto della maledizione colpisca anche uno solo, uno qualunque tra tutti gli individui elencati.

\section{CONCLUSIONI}

Tiriamo a questo punto le fila da quanto l'eccezionale documento permette di osservare. La competenza bilingue del redattore si esercita a livelli 'bassi' di entrambi i sistemi compresenti nel suo repertorio. Certamente la scrittura dell'osco non solo non rispecchia alcun modello ortografico standardizzato, ma presenta perfino all'interno del testo stesso vistose incoerenze. Anche sul versante greco non mancano deviazioni dall'ortografia standard e significative incoerenze interne. Lo sforzo di apporre a sigillo finale del testo un'espressione di maledizione, formalmente ineccepibile, ma altrettanto facilmente disponibile nell'armamentario delle formule magiche, non corrisponde ad un rigore ortografico di pari livello. Allo stesso modo il trattamento dei nomi italici inseriti nella flessione greca mostra incoerenze sia di natura grafica nell'alternare come puri allografi $\langle 0\rangle /\langle\omega\rangle$ senza alcuna apparente logica interna sia di natura linguistica nell'alternare come allomorfi del

102 Ricordiamo che grafie abbreviate di questa particella sono note, ma solo con omissione della parte finale, cioè in ( ), comune nella Tabula Bantina, $\varepsilon \iota v(\varepsilon \iota \mu)$ a Rossano di Vaglio, ini $(m)$ in area campano-sannita.

$103 \mathrm{Su}$ questi valori sviluppati come effetti di senso contestuale dalle marche della coordinazione connettiva in diverse lingue, tra cui quelle sabelliche, cfr. Orlandini-Poccetti 2007, p. 204 ss. 
greco parlato, sociolinguisticamente basso, la flessione dei temi in consonante $-\alpha \kappa-,-v \tau-/-\delta$ -

Il testo sembra, dunque, rispecchiare una condizione in cui osco e greco erano sicuramente compresenti ed attivi nel repertorio sociolinguistico dei parlanti. Va da sé che era sicuramente il greco ad occupava la scala più alta del repertorio, ma collocandosi in un continuum et gradatum rispetto all'osco, come solitamente avviene in situazioni di diglossia.

Il testo della defixio, pur convergendo con il quadro documentario più ampio di Petelia nell'additare la condizione subalterna dell'osco, riservato, per usare un'espressione di G. Berruto, ai «livelli della socializzazione primaria», non conferisce, tuttavia, l'impressione che l'osco sia in una condizione regressiva. Tale circostanza sarebbe, del resto, sorprendente in rapporto alla datazione 'alta' del documento, cioè la fine del IV secolo, epoca che in altre località, fuori e dentro la stessa regione, segna il fiorire dell'epigrafia osca. Dal testo della defixio, si riceve, invece, l'impressione che i due codici vengano rappresentati e quasi equamente ripartiti tanto nelle formule onomastiche (se si accoglie la distinzione tra flessione osca, sicura, e flessione greca, probabile) quanto nell'espressione di maledizione, con l'accortezza di non creare ridondanze o sovrapposizioni. Così, per esempio, vengono ripartite le categorie della flessione, con cui distribuiscono in maniera alquanto sistematica rispettivamente i nominativi all'osco e i genitivi al greco. Inoltre, nella formula di maledizione la parte in osco non è la mera traduzione della sezione in greco. Le due espressioni si complementano e si rinforzano vicendevolmente.

Saremmo pertanto inclini a non estendere a questo documento le stesse categorie interpretative che M.Mancini ha appropriatamente applicato alla tabella defixionis da Cuma, ${ }^{104}$ nella quale effettivamente l'uso dell'osco nella formula magica in un contesto storico e istituzionale ormai latinizzato nel profondo, come denuncia la romanizzazione delle designazioni personali di quel testo, rivela la regressione della lingua locale sotto la pressione della lingua di maggior prestigio, manifestando la marginalizzazione attraverso la sua riduzione a sottocodice in settori o contesti comunicativi subalterni.

104 Cfr. Mancini 1988. Per quanto riguarda il nostro testo si veda Mancini 2012, 253. 
Nella defixio di Petelia, invece, l'osco appare resistere con pari dignità a fianco del greco nelle designazioni personali sia a livello strutturale (la designazione binomia) sia a livello morfologico (tutte le uscite di nominativi in osco sono formalmente ineccepibili anche nella distinzione tra marca del prenome e marca del gentilizio). ${ }^{105}$ Nella formula di maledizione l'osco si affianca al greco senza che le due porzioni interferiscano reciprocamente o si sovrappongano anche parzialmente, quasi a voler segnalare che la lingua con cui si invoca il maleficio rispecchia il pluralismo linguistico del repertorio quotidiano. Sotto tale punto di vista, il testo della defixio è indubbiamente uno degli esempi più straordinari di code-switching e di continuum sociolinguistico che il mondo antico ci metta a disposizione. Tuttavia, lo straordinario documento ci dimostra anche come le categorie del plurilinguismo e del 'mistilinguismo' all'interno non solo di un ambiente urbano, ma anche di ciascun testo siano estremamente graduabili, sfaccettate ed articolate.

In conclusione, nella lingua d'uso, almeno a questo livello cronologico e in questo specifico contesto, l'osco sembra mantenere salde le sue posizioni, pur restando escluso dai livelli della comunicazione alta, come ci mostra il dossier epigrafico della città, in piena sintonia con quello di altri siti bruzi. Più tecnicamente le risultanze di questa analisi appaiono meglio adattarsi alla nozione di "dilalia" enucleata da G.Berruto per l'ambito italofono come «bilinguismo endogeno a bassa distanza strutturale», in cui «l'italiano o un dialetto italo-romanzo è la lingua della socializzazione primaria e l'italiano la lingua della socializzazione secondaria». In realtà, la "dilalia" è uno sviluppo della "diglossia”, per cui la lingua sociolinguisticamente 'alta' tende ad estendersi sempre più anche nei contesti informali, sostituendosi alle varietà 'basse', quale è appunto la situazione sincronica dell'italiano. Ma, ovviamente, non accade l'inverso.

Nel caso di Petelia questi ruoli vengono rispettivamente assunti dal greco e da una varietà di osco (probabilmente con tratti locali e certamente non allineata allo standard dell'osco meridionale), nella quale la distanza strutturale rispetto al greco sembra essersi, in qualche misura, ridotta, come mostrano i casi di induzione di morfemi e di allomorfismi flessionali: fenomeni che si travaseranno nel latino dopo la romanizzazione. L'uso del greco, che fuor di ogni dubbio rivestiva in quella 
società il ruolo di maggior prestigio, anche in un contesto informale, quale quello della formula di maledizione, in cui, però, non è assente l'osco, sembra più appropriatamente iscriversi entro un quadro di "dilalia", nel senso sopra delineato.

L'identità culturale italica della città, segnalata dalle strutture designazioni personali, per quanto riguarda tanto il repertorio quanto la struttura binomia, rimane intatta fino all'età romana, anche se di volta in volta adattata alle varie esigenze della comunicazione 'alta' o di contesti internazionali, a cui si riferiscono i testi nn. 7-10.

Questa conclusione si inserisce perfettamente nel quadro storico più generale sintetizzato da $\mathrm{A}$. Mele per cui «la grecità della Crotoniatide sopravvive alla guerra annibalica, ma in mano brettia». ${ }^{106}$ E' questo il quadro sociolinguistico che tra III e II secolo Ennio e Lucilio ritraggono con la formula sintetica e proverbiale di bilingues Bruttaces, di cui le premesse si costituiscono alla fine del IV secolo a cui risale la tabella defixionis sopra commentata.

106 Citazione da Mele 2007, p.183. 


\section{BIBLIOGRAFIA}

Arena R. 1996, Iscrizioni greche arcaiche di Sicilia e Magna Grecia, IV. Iscrizioni delle colonie achee, Alessandria 1996.

Arena R. 1996, Valori differenziati di alcune lettere nelle diverse tradizioni alfabetiche della Grecia antica, AISN 5, 1983, 197-226.

Arangio Ruiz V., Olivieri A. 1925, Inscriptiones Graecae Siciliae et Infimae Italiae ad ius pertinentes, Milano 1925 (rist. Roma 1965).

Audollent A. 1904, Defixionum Tabellae, Paris 1904.

Bechtel 1917, F. Bechtel, Die historischen Personennamen des Griechischen bis zur Kaiserzeit, Halle 1917.

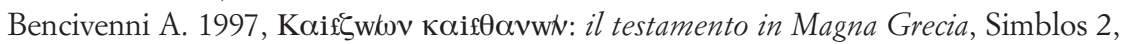
1997, 9-42.

Berruto G. 1996, La varietà del repertorio, in: Introduzione all'italiano contemporaneo. La variazione e gli usi, a cura di A. Sobrero, Roma-Bari 1996, p. 3-36.

Chantraine P. 1933, La formation des noms en grec ancien, Paris 1933.

Cassio A.C. 1985, Old Persian maw kìka-, Eupolis Marikas and Aristophanes Knights, Class. Quat. 35, 1985, 38-42.

Cordiano G. 1997, La ginnasiarchia nelle "poleis" dell'Occidente mediterraneo antico, Pisa 1997.

Costabile F., 1984, Ginnasiarchi a Petelia, ASCL 51, 1984, p. 5-15.

Costabile F. 2007a, Petelia: una polis bruzio-italiota alleata di Roma. Commistione etnica e ibridazione istituzionale, in: Enigmi delle civiltà antiche dal Mediterraneo al Nilo, I, Reggio Calabria 2007, p.365-382.

Costabile F., 2007b, La triplice defixio dal Kerameikos di Atene. Processo e norma libraria attica nel V-IV sec. A.C., in: Enigmi delle civiltà antiche dal Mediterraneo al Nilo, I, Reggio Calabria 2007, p.161-201.

De Sanctis G. 1911, I Thearodokoi di Epidauro, Atti Acc. Scienze Torino 47, 1911-12, pp.442-450 (= Scritti Minori, Roma 1966, I, p.171-179).

De Sensi Sestito 2004, Alessandro e le popolazioni della Lucania e del Bruzio, in: Alessandro il Molosso e i "condottieri" in Magna Grecia, Atti del XLIII convegno di studi sulla Magna Grecia (Taranto-Cosenza 26-30 settembre 2003), Taranto 2004, pp. 519-560.

Dubois L. 1989, Inscriptions grecques dialectales de Sicile, I, Rome 1989.

Dubois L. 2002, Inscriptions grecques dialectales de Grande Grèce II. Colonies Achéennes, Génève 2002.

Dubois L. 2008, Inscriptions grecques dialectales de Sicile, II, Génève 2008.

Dupraz E. 2012, Sabellian Demonstratives. Forms and Functions, Leiden 2012.

Fraser P.M., Matthews E. 1994, A Lexicon of Greek Personal Names. II. Attica, Oxford 1994. 
Gasperini L. 1986, Vecchie e nuove epigrafi del Bruzio ionico, in: Decima Miscellanea Greca e Romana, Roma 1986, pp. 141-171.

Giangiulio M. 1991, Filottete tra Sibari e Crotone. Osservazioni sulla tradizione letteraria, in: Epeios et Philoctète en Italie, Données archéologiques et traditions légendaires, Actes du colloque International (Lille, 23-24 novembre 1987), Naples 1991, pp. 37-53.

Granei D. 1995, La laminetta cosiddetta di Petelia. Per una riconsiderazione dei dati di rinvenimento, Studi di antichità 8,1, 1995, p. 69-76.

Granei D. 1999, La laminetta di Terina: considerazioni ed ipotesi, in: Tra l'Amato e il Savuto, II. Studi sul Lametino antico e tardo-antico, a cura di G. De Sensi Sestito, Soveria Mannelli 1999, 139-157.

Guzzo P.G. 1988, Osservazioni sull'archeologia dei primi Brezi, in: Per un'identità culturale dei Brettii, a cura di P. Poccetti, Napoli 1988, p. 179- 188.

Intrieri M., Zumbo A. 1995, I Brettii. II. Fonti letterarie ed epigrafiche, Soveria Mannelli 1995.

Krahe H. 1941, Die Ortsnamen des antiken Lukanien und Bruttierlandes, ZONF 17, 1941, p.127-150.

Lazzarini M.L.- Lattanzi E. - Mosino F. 1979, La tegola di Pellaro (Reggio Calabria), PP 44, 1989, p. 286-310.

Lazzarini M.L. 1994, Una nuova defixio greca da Tiriolo, in: Forme di religiosità e tradizioni sapienziali in Magna Grecia (Atti del convegno, Napoli, 14-15 dicembre 1993), AION Fil.-Lett. 16, 1994, p. 163-169.

Lazzarini M.L. 2000a, Grecanici come gentilizi. Una nuova iscrizione latina da Petelia,

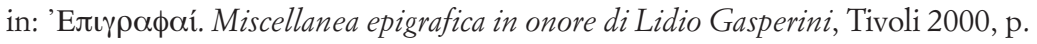
503-510.

Lazzarini 2000b, M.L.Lazzarini, Un nome femminile sibarita, dans Philokypros. Mélanges O. Masson, Suppl. Minos 16, Salamanca 2000, 197-199.

Lazzarini M.L. 2003, L'eponimia a Crotone, A proposito di una nuova laminetta bronzea iscritta. In: Epigraphica. Atti della giornate di studio di Roma e di Atene in memoria di M.Guarducci (1902-1999), Opuscula Epigraphica 10, 2003, 81-88.

Lazzarini M.L. 2004a, Lamina plumbea iscritta da Petelia, Mediterraneo Antico 7, 2004, p. $673-680$.

Lazzarini M.L. 2004b, Pantomimi a Petelia, Arch.Cl. 55, 2004, p. 363-372.

Lazzarini M.L. 2009, Interazioni onomastiche nel Bruzio ionico, in : L'onomastica dell'Italia antica: aspetti linguistici, storici, culturali, tipologici e classificatori, Collection de l'École Française de Rome 413, Roma 2009, p. 425-431.

Lazzarini M.L. 2012, Interazioni culturali tra Greci e Brettii. L'epigrafia di Petelia, in: Enotri e Brettii in Magna Grecia. Modi e forme di interazione culturale, a cura di G. De Sensi Sestito e S.Mancuso, Soveria Mannelli 2012, 595-600.

Lazzeroni R. 1983, Contatti di lingue e culture nell'Italia antica. Modelli egemoni e modelli subordinati nelle iscrizioni osche in grafia greca, AI $\Omega$ N 5, 1983, p. 171-182.

Lazzeroni R. 1994, Contatti di lingue e culture nell'Italia antica: l'onomastica come tramite dell'induzione di morfemi in latino, in: Miscellanea di studi linguistici in onore di W. Belardi, Roma 1994, p. 253-260. 
Lejeune M. 1976, L'anthroponymie osque, Paris 1976.

Lejeune M. 1982, Noms grecs et noms indigènes dans l'épigraphie hellénistique d'Entella, ASNP s. III, 12, 1982, p. 787-799.

Lombardo M. 2001, Enotri e Lucani: continuità e discontinuità, in: Il mondo enotrio tra VI $e$ V secolo a.C.. Atti de seminari napoletani (1996-1998), a cura di M. Bugno e C. Masseria, Napoli, 2001, 329-345.

Mancini M. 1988, Sulla ‘defixio’ osco-latina Vetter 8, SSL 28, 1988, p. 200-230.

Mancini M. 2012, Su alcune questioni di metodo in sociolinguistica storica : le defixiones sannite, in: Per Roberto Gusmani. Linguistica Storica e Teorica, Studi in ricordo, Udine 2012, p. 239-271.

Manganaro G. 1964, Città di Sicilia e santuari panellenici nel III $e$ II secolo a.C., Historia 13,1964, p.414-439.

Mele A. 2007, Magna Grecia. Colonie achee e pitagorismo, Napoli 2007.

Moretti L. 1984, Epigramma per un atleta di Taranto, in: Alessandria e il mondo ellenistico-romano, Studi in onore di A. Adriani, Roma 1984, p. 495-497.

Musti D. 1994, Strabone e la Magna Grecia. Città e popoli dell'Italia antica, Padova 1994.

Musti D. 2005, Magna Grecia. Il quadro storico, Roma-Bari 2005.

Orlandini A., Poccetti P. 2007, Les opérateurs de coordination et les connecteurs en latin et dans d'autres langues de la Méditérranée ancienne, in: La coordination, (sous la direction de A.Rousseau, L. Begioni, N. Quayle, D. Rouilland), Rennes 2007, p. 189-224.

Penney, J. 2002. Notes on some Sabellic Demonstratives. In (I.J. Hartmann \& A. Willi eds.), Oxford University Working Papers in Linguistics, Philology \& Phonetics, 7, 2002, p. 131-142.

Pouilloux J. 1960, Choix d'inscriptions grecques, Textes, traductions et notes, Paris 1960.

Pugliese Carratelli G. 2001, Le lamine d'oro orfiche. Istruzioni per il viaggio oltremondano degli iniziati greci, Milano 2001.

Poccetti P. 1988, Lingua e cultura dei Brettii, in: Per un'identità culturale dei Bretti, a cura di P. Poccetti, Napoli 1988, p. 7-158.

Poccetti P. 1989, Le popolazioni anelleniche d'Italia tra Sicilia e Magna Grecia nel IV secolo a.C.: forme di contatto linguistico e di interazione culturale, in: Atti del convegno Tra Sicilia e Magna Grecia.Aspetti di interazione culturale nel IV sec. a.C. (Napoli 19-20 marzo 1987), AIQN 11, 1989, 97-135.

Poccetti P. 2006, Fenomeni di allomorfismo flessionale in ambiente bilingue osco-greco. Un contributo alla storia di lat. Aī̄x, Bruttāx e oltre, in: Scritti linguistici in onore di Roberto Gusmani, Alessandria 2006, 1359-1372.

Prosdocimi A.L. 1978, Il lessico istituzionale italico tra linguistica e storia, in : La cultura italica, Atti del convegno della Società Italiana di Glottologia (Pisa 19-20 dicembre 1977), Pisa 1978, p. 29-74.

Prosdocimi A.L. 2004, Filoni indoeuropei in Italia. Riflessioni e appunti, in: Scritti inediti e sparsi, Padova 2004, II, p. 1359-1531.

Rix H. 2002, Sabellische Texte, Heidelberg 2002.

Solin H. 1982, Appunti sull'onomastica romana a Delo, in: Delo e l'Italia, Opuscula Instituti Romani Finlandiae II, 1982, p.101-117. 
Tagliamonte G. 1994, I figli di Marte. Mobilità, mercenari e mercenariato in Magna Grecia e Sicilia, Roma 1994.

Tortorelli Ghidini M. 2006, Figli della terra e del cielo stellato, Napoli 2006.

Trendall A.D. 1967, The Red-Figured vases of Lucania, Campania and Sicily, Oxford 1967.

Wikén E. 1937, Die Kunde der Hellenen von dem Lande und den Völkern der Apenninenhalbinsel bis 300. v.Chr., Lund 1936.

Wünsch R. 1897, Inscriptiones Graecae III, 3, Appendix. Defixionum Tabellae, Berlin 1897.

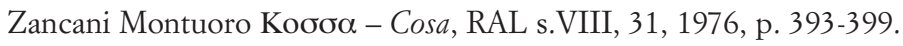




\section{APPENDICE}

\section{TESTI}

1a)

Bilingues Bruttaces Ennius dixit, quod Bruttii et Osce et Graece loqui soliti sint. Sunt autem Italiae populi vicini Lucanis (P. Fest 31 L. = Enn., ann. 496 Vahlen).

1b)

Canusini more bilinguis. Bilinguis dixit quoniam utraque lingua usi sunt, sicut per omnem illum tractum Italiae, quoniam ex maiore parte Graeci ibi incoluerunt ex quo Magnae Graeciae nomen accepit. Ideo ergo et Ennius et Lucilius Bruttace bilingui dixerunt (Porph. Ad Hor., Sat. I 10, 30 = Lucil. 1124 Marx).

2)

Pethlia mé ou mhtropolij nomizetai twa Leukanwa kaifs unoikeikai mekri nu ikanwj. Ktis ma d estiffilokthtou, fugohtoj thr Mel iboian kataf stakin. Erumnhed esti/ wste kaif Saunika i pote frouribij epeteikis a n a uthh (Strab. VI 1,3254 C).

3)

Qeof tuka. Sawtij didoti Sikainiai tah Foikikn kaiftakl a panta. Da miorgof Paragoraj. Prokenoi M inkwn, Armocida moj, A gaqarxoj, Dn a taj, Epikōoj (IG XIV 637; Arena 1996, n 51; Dubois 2002, n 93)

4)

Eurh\$s eij d IA i \#a o domwn ep la ris tera fkrhhhn, paf d duthisleukh自 elthkui n kuparisson:

tauthj thjk khhhj mhdefs xedor empel a $k$ eiaj.

euthseij d leteran, thF M nhmosuhhj a pofl imnhj

y uxrof udwr prorebn: ful akej d lepiprosqen elis in.

eipein: «GhF paif eimi kaifoura nou $=$ \$ $\$$ teroentoj,

a utaf emoifgehoj ourahion: toded libte ka ifautoil

diy hi d eimifauk ka ifapoll umai: a l I a fdot la iy a

y uxrof ußdwr prorebn thj M nhmosuhhj a pofl imnhj».

Ka ut[oif s[0]i dw\$ous i piein=qeihj a p[ofl im]nhj,

ka iftot lepeit pa ll I ois i meq I hitwes s in a hakei[j].

[M nhmosulnhj tode iproh: epeifa mell hisi]

va neisq [a i ? t]ode gra [

(Testo: Tortorelli Ghidini 2006, p .66; cfr. anche IG XIV 638; Pugliese Carratelli 2001, p. 67). 
5)

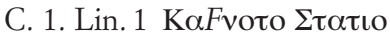

2 П $\alpha \kappa F \iota \omega$ K $\alpha \iota \alpha \delta \omega$

3 Пакод $\Sigma \tau \alpha \tau \iota \varepsilon \varsigma$

4 Mapas $\Sigma \tau \alpha \tau \iota \varepsilon \varsigma$

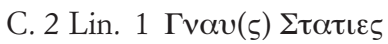

$2 F_{\iota} \beta \mathrm{t}(\varsigma) \Sigma \tau \alpha \tau \iota \varepsilon \varsigma$

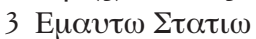

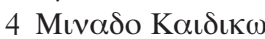

$5 \tau \rho \varepsilon \delta \omega \alpha v \delta \alpha \delta o$

6 Mıvas Kaıঠıкıs

C. 3 Lin. 1 AFes Avdoıs

2 NoFı A $\lambda \alpha \phi \iota \omega$

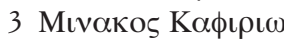

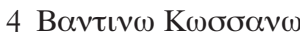

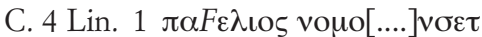

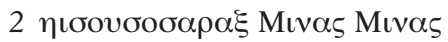

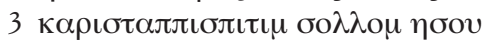

4 dekeo Herma =x qohie ta uka ka ifka geke a utek

(Lazzarini 2004; 2009)

6a)

Eubd[e] / xa ike (IG XIV 639)

6b)

pa [---] / xa ike (IG XIV 640)

7)

[Epifgum[n]asiatx[wn]/ [M inato]u Kri[t]tib[u] M ina[tou] M enidaj (o M atillaj), M a tkou Kri[ttibu]/ [M inat]ou h(stoa faheskeu/a 5 gh ek tõn k o in[w a] xrhmatw n (IG XIV 637; Intrieri-Zumbo 1995, p. 266, B4; Costabile 1984; 2007a, p. 369)

8)

Delo: II sec. a.C.

'A ga qwn N umy ibu P ethl inoj (Intrieri-Zumbo 1995, p.266, B4; IG XI 41244 1246)

9)

Delfi : lista dei thearodokoi

em P ethli i i 'O f a ll i oj (Manganaro 1964, p.420: col. IV 86) 
10)

'E pifl eukipu Or / ka ifN ouibu Ele

Dh (mosion)

(Intrieri-Zumbo 1995, p. 269 B-14; Costabile 2007, p. 365)

11)

Celadioni grex Ionici pantomimi.Vix (it) an(nis) V

Ei) qahaton tog a wron a hacion hipase PI outwn I upaj a enapuj patrif xarica menoj. P ou =s e quein, K el a de; pou=d) aytia bw moif stemmata, suf I iba hw ?q la gna ff er ein pel a na / Tó mogeró s khnhF ekeitpa se batbaroj A [i]dhj, poll a fgoneusi fetwn dakkrua sur pagesin.

(Lazzarini 2004b)

12)

$D($ is) $M$ (anibus) [s(acrum) $]$

T.Bathil[i]o Me/trobio Vibia / Heuresis fecit / coniugi meren/tissimo. Vixit / annis XXX-

VII (Lazzarini 2000).

13)

Have Megonia Euhodis vixit ann(os) XXVI S(it) T(ibi) T(erra) L(evis) (CIL X 8087; AE $84,272)$

14)

Octaviae Crotonidi Sex(ti) Arelli Ursi coniugi merentissimae locus datus

(Gasperini 1986, p. 153-154) 